\title{
AVERAGES AND COMPACT, ABSOLUTELY SUMMING AND NUCLEAR OPERATORS ON $C(\Omega)$
}

\author{
Dumitru Popa
}

\begin{abstract}
In the paper we introduce averages of each type and use these averages to construct examples of weakly compact operators on the space $C(\Omega)$ for which the necessary and sufficient conditions that they be compact, absolutely summing or nuclear are distinct. A great number of concrete examples, in various situations, are given.
\end{abstract}

\section{Introduction}

Let $\Omega$ be a compact Hausdorff space, $\Sigma_{\Omega}$ the $\sigma$-field of Borel subsets of $\Omega$, $C(\Omega)$ the space of all scalar-valued continuous functions on $\Omega$ under the uniform norm, $X$ a Banach space and $U: C(\Omega) \rightarrow X$ a bounded linear operator. It is well-known, see [3, Chapter VI], that $U$ has a representing vector measure $G$, and that $U$ is weakly compact if and only if $G$ takes its values in $X$; $U$ is compact if and only if $G$ has norm compact range; $U$ is absolutely summing if and only if $G$ has bounded variation; $U$ is nuclear if and only if $G$ has a Bochner integrable Radon-Nikodym derivative with respect to its variation $|G|$.

In [8] are given explicit examples of bounded linear operators on $C[0,1]$ with values in $c_{0}$ which distinguish certain ideals of operators. In this paper we complete the results and examples in [8] by giving many other examples.

We fix now some notations and terminology. Let $X$ be a Banach space, $\Sigma$ a $\sigma$-field of sets and $G: \Sigma \rightarrow X$ a vector measure. We denote by $|G|$ the variation measure of $G,\|G\|$ the semivariation, $\|G\|(E)=\sup _{\left\|x^{*}\right\| \leq 1}\left|x^{*} G\right|(E), E \in \Sigma$, see [3, Chapter I, pp. 3-4]. If $(S, \Sigma, \mu)$ is a finite measure space, $X$ a Banach space and $f: S \rightarrow X$ a $\mu$-Bochner integrable function we write $\int_{(\cdot)} f d \mu$ for the Bochner integral; if $f: S \rightarrow X$ is a $\mu$-Pettis integrable function, the Pettis norm of $f$ is defined by $\|f\|_{\text {Pettis }}=\sup _{\left\|x^{*}\right\| \leq 1} \int_{S}\left|x^{*} f\right| d \mu$, see [3, Chapter II].

If $\left(X_{n}\right)_{n \in \mathbb{N}}$ is a sequence of Banach spaces, we denote $c_{0}\left(X_{n} \mid n \in \mathbb{N}\right)$, the Banach space of all sequences $\left(x_{n}\right)_{n \in \mathbb{N}}, x_{n} \in X_{n}$ for every $n \in \mathbb{N}$, $\left\|x_{n}\right\| \rightarrow 0$, endowed to the norm $\left\|\left(x_{n}\right)_{n \in \mathbb{N}}\right\|=\sup _{n \in \mathbb{N}}\left\|x_{n}\right\|$ and similarly, $l_{\infty}\left(X_{n} \mid n \in \mathbb{N}\right)$

Received June 20, 2008; Revised January 6, 2010.

2000 Mathematics Subject Classification. 46B28, 47B10, 47G10.

Key words and phrases. Banach spaces of continuous functions, weakly compact, compact, nuclear, $p$-summing. 
denote the Banach space of all sequences $\left(x_{n}\right)_{n \in \mathbb{N}}, x_{n} \in X_{n}$ for every $n \in \mathbb{N}$, with $\sup _{n \in \mathbb{N}}\left\|x_{n}\right\|<\infty$, endowed to the norm $\left\|\left(x_{n}\right)_{n \in \mathbb{N}}\right\|=\sup _{n \in \mathbb{N}}\left\|x_{n}\right\|$.

When $X_{n}=X$, we write $c_{0}(X)$ resp. $l_{\infty}(X)$. By $l_{\infty}^{n}(X)$ we denote

$$
(\underbrace{X \times \cdots \times X}_{n \text { times }},\|\|_{\infty}) .
$$

The scalar field $\mathbb{R}$ (or $\mathbb{C}$ ) is denoted $\mathbb{K}$ and if $n \in \mathbb{N}, 1 \leq p \leq \infty$, then

$$
l_{p}^{n}=\left(\mathbb{K}^{n},\|\|_{p}\right)
$$

where $\left\|\left(\alpha_{1}, \ldots, \alpha_{n}\right)\right\|_{p}=\left(\sum_{i=1}^{n}\left|\alpha_{i}\right|^{p}\right)^{\frac{1}{p}}$ if $p<\infty$ and $\left\|\left(\alpha_{1}, \ldots, \alpha_{n}\right)\right\|_{\infty}=$ $\max _{1 \leq i \leq n}\left|\alpha_{i}\right|$. Further $p^{*}$ is the conjugate of $p$ and by $\left(e_{n i}\right)_{1 \leq i \leq n}$ we denote the standard basis in $l_{p}^{n}$.

If $\left(a_{n}\right)_{n \in \mathbb{N}},\left(b_{n}\right)_{n \in \mathbb{N}}$ are two real sequences we write $a_{n} \asymp b_{n}$ if and only if there exist $m, M>0$ such that $m b_{n} \leq a_{n} \leq M b_{n}$ for every $n \in \mathbb{N}$. If $k \in \mathbb{N}$ and $\left(a_{n k}\right)_{n \in \mathbb{N}},\left(b_{n k}\right)_{n \in \mathbb{N}}$ are two real sequences we write $a_{n k} \asymp b_{n k}$ if and only if there exist $m_{k}, M_{k}>0$ such that $m_{k} b_{n k} \leq a_{n k} \leq M_{k} b_{n k}$ for every $n \in \mathbb{N}$.

If $X$ is a Banach space, $1 \leq p<\infty, m \in \mathbb{N}$ and $x_{1}, x_{2}, \ldots, x_{m}$ a finite system of vectors in $X$, we write

$$
\begin{aligned}
w_{p}\left(x_{i} \mid 1 \leq i \leq m ; X\right) & =\sup _{\left\|x^{*}\right\| \leq 1}\left(\left|x^{*}\left(x_{1}\right)\right|^{p}+\cdots+\left|x^{*}\left(x_{m}\right)\right|^{p}\right)^{\frac{1}{p}} \\
& =\left\|T: X^{*} \rightarrow l_{p}^{m}\right\|
\end{aligned}
$$

where $T\left(x^{*}\right)=\left(x^{*}\left(x_{1}\right), \ldots, x^{*}\left(x_{m}\right)\right)$.

In the rest of the paper, $\mathcal{B}$ denotes the $\sigma$-algebra of all Borel sets in $[0,1]$, $\lambda: \mathcal{B} \rightarrow[0,1]$ the Lebesgue measure, $\left(r_{n}\right)_{n \in \mathbb{N}}$ the sequence of Rademacher functions and $C[0,1]$ the space of all scalar-valued continuous functions on $[0,1]$ under the uniform norm. If $X$ is a Banach space, $L_{1}(\lambda, X)$ is the space of $\lambda$-Bochner integrable functions. If $\mu, \nu$ are two positive measures we denote $\mu \otimes \nu$ their product.

All notation and terminology, not otherwise explained, are as in $[2,3]$.

\section{Scalar and vector averages}

Let $X$ be a Banach space, $m \in \mathbb{N}$ and $x_{1}, x_{2}, \ldots, x_{m}$ a finite system of vectors in $X$. As in [8] we define Average $\left(x_{i} \mid 1 \leq i \leq m\right)$ as the finite system with $2^{m}$ elements obtained by arranging in the lexicographical order of $\{-1,1\}^{m}$, the set of all the elements of the form $\varepsilon_{1} x_{1}+\cdots+\varepsilon_{m} x_{m}$ for $\left(\varepsilon_{1}, \ldots, \varepsilon_{m}\right) \in$ $\{-1,1\}^{m}=D_{m}$ (On $\{-1,1\}$ we consider the natural order). We will consider Average $\left(x_{i} \mid 1 \leq i \leq m\right)$ as an element of the space $X^{2^{m}}$ and as sets we have the equality

$$
\text { Average }\left(x_{i} \mid 1 \leq i \leq m\right)=\left\{\varepsilon_{1} x_{1}+\cdots+\varepsilon_{m} x_{m} \mid\left(\varepsilon_{1}, \ldots, \varepsilon_{m}\right) \in D_{m}\right\} .
$$


The idea of considering these averages was suggested to the author by the well-known discrete form of Rademacher means, namely the equality

$$
\int_{0}^{1}\left\|x_{1} r_{1}(t)+\cdots+x_{m} r_{m}(t)\right\| d t=\frac{1}{2^{m}} \sum_{\left(\varepsilon_{1}, \ldots, \varepsilon_{m}\right) \in D}\left\|\varepsilon_{1} x_{1}+\cdots+\varepsilon_{m} x_{m}\right\|
$$

see [1], [2]. Further, in [1, Exercise 8.18(a), p. 107], or [9, p. 64] appear also these averages.

Lemma 1. Let $m \in \mathbb{N}$ and $\alpha_{1}, \alpha_{2}, \ldots, \alpha_{m}$ be a finite system of scalars. Then

$$
\begin{gathered}
\| \text { Average }\left(\alpha_{i} \mid 1 \leq i \leq m\right)\left\|_{\infty} \asymp\right\|\left(\alpha_{1}, \ldots, \alpha_{m}\right) \|_{1}, \\
\| \text { Average }\left(\alpha_{i} \mid 1 \leq i \leq m\right)\left\|_{1} \asymp 2^{m}\right\|\left(\alpha_{1}, \ldots, \alpha_{m}\right) \|_{2}, \\
\| \text { Average }\left(\alpha_{i} \mid 1 \leq i \leq m\right)\left\|_{2}=(\sqrt{2})^{m}\right\|\left(\alpha_{1}, \ldots, \alpha_{m}\right) \|_{2} .
\end{gathered}
$$

Proof. Indeed, in the real case, we have obvious

$$
\| \text { Average }\left(\alpha_{i} \mid 1 \leq i \leq m\right) \|_{\infty}=\max _{\left(\varepsilon_{1}, \ldots, \varepsilon_{m}\right) \in D_{m}}\left|\varepsilon_{1} \alpha_{1}+\cdots+\varepsilon_{m} \alpha_{m}\right|=\sum_{i=1}^{m}\left|\alpha_{i}\right|
$$

and from here, taking the real and imaginary part, we deduce, in the complex case

$$
\frac{1}{2} \sum_{i=1}^{m}\left|\alpha_{i}\right| \leq \| \text { Average }\left(\alpha_{i} \mid 1 \leq i \leq m\right) \|_{\infty} \leq \sum_{i=1}^{m}\left|\alpha_{i}\right|
$$

see also [1, Exercise 8.18(a), p. 107], or [9, p. 64].

For the second, by Khichin's inequality, see [1], [2], [5], we have $\|$ Average $\left(\alpha_{i} \mid 1 \leq i \leq m\right)\left\|_{1}=\sum_{\varepsilon \in D_{m}}\left|\varepsilon_{1} \alpha_{1}+\cdots+\varepsilon_{m} \alpha_{m}\right| \asymp 2^{m}\right\|\left(\alpha_{1}, \ldots, \alpha_{m}\right) \|_{2}$.

The last equality follows from the well-known equality

$$
\begin{aligned}
\| \text { Average }\left(\alpha_{i} \mid 1 \leq i \leq m\right) \|_{2} & =\left(\sum_{\varepsilon \in D_{m}}\left|\varepsilon_{1} \alpha_{1}+\cdots+\varepsilon_{m} \alpha_{m}\right|^{2}\right)^{\frac{1}{2}} \\
& =(\sqrt{2})^{m}\left\|\left(\alpha_{1}, \ldots, \alpha_{m}\right)\right\|_{2} .
\end{aligned}
$$

Our next definition is a natural iteration for averages.

Definition 2. For $k \in \mathbb{N} \bigcup\{0\}$ define $f_{k}: \mathbb{N} \rightarrow \mathbb{N}$ by

$$
\left\{\begin{array}{l}
f_{0}(n)=n \\
f_{k+1}(n)=2^{f_{k}(n)}, k \geq 0
\end{array}\right.
$$

Let $X$ be a Banach space, $n \in \mathbb{N}$ and $x_{1}, x_{2}, \ldots, x_{n}$ a finite system of vectors in $X$. Define

$$
\text { Average }_{1}\left(x_{i} \mid 1 \leq i \leq n ; X\right)=\operatorname{Average}\left(x_{i} \mid 1 \leq i \leq n ; X\right) .
$$


Let also $k \in \mathbb{N}$. For the $f_{k}(n)$ finite system

$$
\operatorname{Average}_{k}\left(x_{i} \mid 1 \leq i \leq n ; X\right)=\left\{\beta_{1}, \ldots, \beta_{f_{k}(n)}\right\},
$$

say, we apply the same procedure and denote

$$
\text { Average }_{k+1}\left(x_{i} \mid 1 \leq i \leq n ; X\right)=\operatorname{Average}\left(\beta_{i} \mid 1 \leq i \leq f_{k}(n) ; X\right) .
$$

We consider Average $_{k}\left(x_{i} \mid 1 \leq i \leq n ; X\right)$ as an element of the space $X^{f_{k}(n)}$.

Lemma 3. Let $n \in \mathbb{N}, \alpha_{1}, \alpha_{2}, \ldots, \alpha_{n}$ be a finite system of scalars and $k \in \mathbb{N}$. Then

$$
\begin{aligned}
& \| \text { Average }\left(\alpha_{i} \mid 1 \leq i \leq n\right)\left\|_{\infty} \asymp\right\|\left(\alpha_{1}, \ldots, \alpha_{n}\right) \|_{1} \text {, } \\
& \| \text { Average }_{2}\left(\alpha_{i} \mid 1 \leq i \leq n\right)\left\|_{\infty} \asymp 2^{n}\right\|\left(\alpha_{1}, \ldots, \alpha_{n}\right) \|_{2} \text {, } \\
& \| \text { Average }_{k}\left(\alpha_{i} \mid 1 \leq i \leq n\right) \|_{\infty} \\
& \asymp f_{k-1}(n) \sqrt{f_{1}(n) f_{2}(n) \cdots f_{k-2}(n)}\left\|\left(\alpha_{1}, \ldots, \alpha_{n}\right)\right\|_{2}, k \geq 3 .
\end{aligned}
$$

Proof. With the same notations as in Definition 2, by Lemma 1 we have

$$
\begin{aligned}
& \| \text { Average }_{k+1}\left(\alpha_{i} \mid 1 \leq i \leq n\right)\left\|_{\infty}=\right\| \text { Average }\left(\beta_{i} \mid 1 \leq i \leq f_{k}(n)\right) \|_{\infty} \\
\asymp & \left\|\left(\beta_{1}, \ldots, \beta_{f_{k}(n)}\right)\right\|_{1}=\| \text { Average }_{k}\left(\alpha_{i} \mid 1 \leq i \leq n\right) \|_{1}, \\
& \| \text { Average }_{k+1}\left(\alpha_{i} \mid 1 \leq i \leq n\right)\left\|_{1}=\right\| \text { Average }\left(\beta_{i} \mid 1 \leq i \leq f_{k}(n)\right) \|_{1} \\
\asymp & 2^{f_{k}(n)}\left\|\left(\beta_{1}, \ldots, \beta_{f_{k}(n)}\right)\right\|_{2}=f_{k+1}(n) \| \text { Average }_{k}\left(\alpha_{i} \mid 1 \leq i \leq n\right) \|_{2}
\end{aligned}
$$

and

$$
\begin{aligned}
\| \text { Average }_{k+1}\left(\alpha_{i} \mid 1 \leq i \leq n\right) \|_{2} & =\| \text { Average }\left(\beta_{i} \mid 1 \leq i \leq f_{k}(n)\right) \|_{2} \\
& =\sqrt{2^{f_{k}(n)}}\left\|\left(\beta_{1}, \ldots, \beta_{f_{k}(n)}\right)\right\|_{2} \\
& =\sqrt{f_{k+1}(n)} \| \text { Average }_{k}\left(\alpha_{i} \mid 1 \leq i \leq n\right) \|_{2} .
\end{aligned}
$$

Denote

$$
\begin{aligned}
a_{k} & =\| \text { Average }_{k}\left(\alpha_{i} \mid 1 \leq i \leq n\right) \|_{\infty}, \\
b_{k} & =\| \text { Average }_{k}\left(\alpha_{i} \mid 1 \leq i \leq n\right) \|_{1}, \\
c_{k} & =\| \text { Average }_{k}\left(\alpha_{i} \mid 1 \leq i \leq n\right) \|_{2} .
\end{aligned}
$$

Then from the above proved relations for each $k \geq 1$ we have

$$
a_{k+1} \asymp b_{k} ; b_{k+1} \asymp f_{k+1}(n) c_{k} ; c_{k+1}=c_{k} \sqrt{f_{k+1}(n)} .
$$

Because by Lemma 1

$$
c_{1}=\| \text { Average }\left(\alpha_{i} \mid 1 \leq i \leq n\right)\left\|_{2}=\sqrt{f_{1}(n)}\right\|\left(\alpha_{1}, \ldots, \alpha_{n}\right) \|_{2}
$$

we deduce

$$
c_{k}=\sqrt{f_{1}(n) f_{2}(n) \cdots f_{k}(n)}\left\|\left(\alpha_{1}, \ldots, \alpha_{n}\right)\right\|_{2}, k \geq 1 .
$$


AVERAGES, COMPACT, ABSOLUTELy SUMMing AND NUClEAR OPERATORS 903

From $b_{k+1} \asymp f_{k+1}(n) c_{k}$ we get

$$
b_{k+1} \asymp f_{k+1}(n) \sqrt{f_{1}(n) f_{2}(n) \cdots f_{k}(n)}\left\|\left(\alpha_{1}, \ldots, \alpha_{n}\right)\right\|_{2}, k \geq 1,
$$
i.e.,

$$
b_{k} \asymp f_{k}(n) \sqrt{f_{1}(n) f_{2}(n) \cdots f_{k-1}(n)}\left\|\left(\alpha_{1}, \ldots, \alpha_{n}\right)\right\|_{2}, k \geq 2,
$$

and by Lemma 1

$$
b_{1}=\| \text { Average }\left(\alpha_{i} \mid 1 \leq i \leq n\right)\left\|_{1} \asymp 2^{n}\right\|\left(\alpha_{1}, \ldots, \alpha_{n}\right) \|_{2} .
$$

From $a_{k+1} \asymp b_{k}, k \geq 1$ we get

$$
a_{k+1} \asymp f_{k}(n) \sqrt{f_{1}(n) f_{2}(n) \cdots f_{k-1}(n)}\left\|\left(\alpha_{1}, \ldots, \alpha_{n}\right)\right\|_{2}, k \geq 2,
$$

i.e., for $k \geq 3$ we get the evaluations from the statement.

Also by Lemma 1,

$$
\begin{aligned}
& a_{2} \asymp b_{1}=2^{n}\left\|\left(\alpha_{1}, \ldots, \alpha_{n}\right)\right\|_{2}, \\
& a_{1}=\| \text { Average }\left(\alpha_{i} \mid 1 \leq i \leq n\right)\left\|_{\infty} \asymp\right\|\left(\alpha_{1}, \ldots, \alpha_{n}\right) \|_{1} .
\end{aligned}
$$

We state now a result which is a well-known consequence of the Hahn-Banach theorem.

Result. Let $X$ be a Banach space. Then for each $x \in X$ we have

$$
\|x\|=\sup _{\left\|x^{*}\right\| \leq 1}\left|x^{*}(x)\right| .
$$

Lemma 4. Let $X$ be a Banach space, $n \in \mathbb{N}, x_{1}, x_{2}, \ldots, x_{n}$ a finite system of vectors in $X$ and $k \in \mathbb{N}$. Then

$$
\begin{aligned}
& \| \text { Average }\left(x_{i} \mid 1 \leq i \leq n ; X\right) \|_{\infty} \asymp w_{1}\left(x_{i} \mid 1 \leq i \leq n ; X\right), \\
& \| \text { Average }_{2}\left(x_{i} \mid 1 \leq i \leq n ; X\right) \|_{\infty} \asymp 2^{n} w_{2}\left(x_{i} \mid 1 \leq i \leq n ; X\right) \text {, } \\
& \| \text { Average }_{k}\left(x_{i} \mid 1 \leq i \leq n ; X\right) \|_{\infty} \\
& \asymp f_{k-1}(n) \sqrt{f_{1}(n) f_{2}(n) \cdots f_{k-2}(n)} w_{2}\left(x_{i} \mid 1 \leq i \leq n ; X\right), k \geq 3 .
\end{aligned}
$$

Proof. We will use the notations from Definition 2. From Result we have

$$
\begin{aligned}
& \| \text { Average }\left(x_{i} \mid 1 \leq i \leq n ; X\right) \|_{\infty} \\
= & \max _{\varepsilon \in D_{n}}\left\|\varepsilon_{1} x_{1}+\varepsilon_{2} x_{2}+\cdots+\varepsilon_{n} x_{n}\right\| \\
= & \max _{\varepsilon \in D_{n}} \sup _{\left\|x^{*}\right\| \leq 1}\left|\varepsilon_{1} x^{*}\left(x_{1}\right)+\varepsilon_{2} x^{*}\left(x_{2}\right)+\cdots+\varepsilon_{n} x^{*}\left(x_{n}\right)\right| \\
= & \sup _{\left\|x^{*}\right\| \leq 1} \max _{\varepsilon \in D_{n}}\left|\varepsilon_{1} x^{*}\left(x_{1}\right)+\varepsilon_{2} x^{*}\left(x_{2}\right)+\cdots+\varepsilon_{n} x^{*}\left(x_{n}\right)\right| \\
= & \sup _{\left\|x^{*}\right\| \leq 1} \| \text { Average }\left(x^{*}\left(x_{i}\right) \mid 1 \leq i \leq n\right) \|_{\infty} .
\end{aligned}
$$

By Lemma 1, for each $\left\|x^{*}\right\| \leq 1$ we have

$$
\| \text { Average }\left(x^{*}\left(x_{i}\right) \mid 1 \leq i \leq n\right)\left\|_{\infty} \asymp\right\|\left(x^{*}\left(x_{i}\right) \mid 1 \leq i \leq n\right) \|_{1}
$$


thus

$$
\| \text { Average }\left(x_{i} \mid 1 \leq i \leq n ; X\right) \|_{\infty} \asymp w_{1}\left(x_{i} \mid 1 \leq i \leq n ; X\right) .
$$

We prove now that for each $k \geq 1$,

$\|$ Average $_{k+1}\left(x_{i} \mid 1 \leq i \leq n ; X\right)\left\|_{\infty} \asymp \sup _{\left\|x^{*}\right\| \leq 1}\right\|$ Average $_{k}\left(x^{*}\left(x_{i}\right) \mid 1 \leq i \leq n\right) \|_{1}$.

Indeed, by Lemma 1 and from what we have proved above we deduce

$$
\begin{aligned}
& \| \text { Average }_{k+1}\left(x_{i} \mid 1 \leq i \leq n ; X\right) \|_{\infty} \\
= & \| \text { Average }\left(\beta_{i} \mid 1 \leq i \leq f_{k}(n) ; X\right) \|_{\infty} \\
\asymp & w_{1}\left(\beta_{i} \mid 1 \leq i \leq f_{k}(n) ; X\right) \\
= & \sup _{\left\|x^{*}\right\| \leq 1}\left\|\left(x^{*}\left(\beta_{i}\right) \mid 1 \leq i \leq f_{k}(n)\right)\right\|_{1} \\
= & \sup _{\left\|x^{*}\right\| \leq 1} \| \text { Average }_{k}\left(x^{*}\left(x_{i}\right) \mid 1 \leq i \leq n\right) \|_{1} .
\end{aligned}
$$

The Lemma 3, implies, for each $k \geq 2$ and each $\left\|x^{*}\right\| \leq 1$

$$
\begin{aligned}
& \| \text { Average }_{k}\left(x^{*}\left(x_{i}\right) \mid 1 \leq i \leq n\right) \|_{1} \\
\asymp & f_{k}(n) \sqrt{f_{1}(n) f_{2}(n) \cdots f_{k-1}(n)}\left\|\left(x^{*}\left(x_{1}\right), \ldots, x^{*}\left(x_{n}\right)\right)\right\|_{2} .
\end{aligned}
$$

Hence for $k \geq 2$

$$
\begin{aligned}
& \| \text { Average }_{k+1}\left(x_{i} \mid 1 \leq i \leq n ; X\right) \|_{\infty} \\
\asymp & f_{k}(n) \sqrt{f_{1}(n) f_{2}(n) \cdots f_{k-1}(n)} w_{2}\left(x_{i} \mid 1 \leq i \leq n ; X\right)
\end{aligned}
$$

i.e., for $k \geq 3$ we get the evaluations from the statement.

Also, from Lemma 1

$$
\begin{aligned}
\| \text { Average }_{2}\left(x_{i} \mid 1 \leq i \leq n ; X\right) \|_{\infty} & \asymp \sup _{\left\|x^{*}\right\| \leq 1} \| \text { Average }\left(x^{*}\left(x_{i}\right) \mid 1 \leq i \leq n\right) \|_{1} \\
& \asymp 2^{n} \sup _{\left\|x^{*}\right\| \leq 1}\left\|\left(x^{*}\left(x_{i}\right) \mid 1 \leq i \leq n\right)\right\|_{2} \\
& =2^{n} w_{2}\left(x_{i} \mid 1 \leq i \leq n ; X\right) .
\end{aligned}
$$

Notation. Let $\left(X_{n}\right)_{n \in \mathbb{N}}$ be a sequence of Banach spaces, $\left(x_{n i}\right)_{1 \leq i \leq n} \subset X_{n}$ for each $n \in \mathbb{N}$. For each $n \in \mathbb{N}$, Average $\left(x_{n i} \mid 1 \leq i \leq n ; X_{n}\right)$ is an element of the space $X_{n}^{2^{n}}$ and we consider the sequence

$$
\text { (Average } \left.\left(x_{n i} \mid 1 \leq i \leq n ; X_{n}\right)\right)_{n \in \mathbb{N}} .
$$

From Lemma 4 , the sequence $(*)$ is an element of the space

$$
c_{0}\left(X_{1}, X_{1}, \ldots, X_{n}, \ldots, X_{n}, \ldots\right)
$$

(each $X_{n}$ appears $2^{n}$ for each $\left.n \in \mathbb{N}\right)$ if and only if $w_{1}\left(x_{n i} \mid 1 \leq i \leq n ; X_{n}\right) \rightarrow 0$.

In order to avoid unpleasant writings, instead $(* *)$ we write simply $c_{0}\left(X_{n}\right)$ $n \in \mathbb{N}$ ). 
AVERAGES, COMPACT, ABSOLUTELY SUMMING AND NUCLEAR OPERATORS 905

In the rest of the paper for a natural number $k \geq 2$ we denote

$$
\begin{aligned}
& b_{n 2}=2^{n}, \\
& b_{n k}=f_{k-1}(n) \sqrt{f_{1}(n) f_{2}(n) \cdots f_{k-2}(n)}, \text { if } k \geq 3 .
\end{aligned}
$$

Using the same convention as above, from Lemma 4 , the sequence

$\left(\text { Average }_{k}\left(x_{n i} \mid 1 \leq i \leq n ; X_{n}\right)\right)_{n \in \mathbb{N}}$ is an element of the space $c_{0}\left(X_{n} \mid n \in \mathbb{N}\right)$ if and only if $b_{n k} w_{2}\left(x_{i} \mid 1 \leq i \leq n ; X_{n}\right) \rightarrow 0$.

\section{The main results}

We begin with a well-known fact:

Fact. Let $(S, \Sigma, \mu)$ be a finite measure space, $X, Y$ Banach spaces, $g: S \rightarrow$ $L(X, Y)$ a $\mu$-Bochner integrable function and $G: \Sigma \rightarrow L(X, Y)$,

$$
G(E)=\int_{E} g d \mu \text { for } E \in \Sigma \text {. }
$$

Then

$$
\|g\|_{\text {Pettis }}=\|G\|(T)=\sup _{\|x\| \leq 1,\left\|y^{*}\right\| \leq 1} \int_{S}\left|\left\langle g(s) x, y^{*}\right\rangle\right| d \mu(s) .
$$

This follows from the definition of semivariation and the Pettis norm and the fact that $\left\{x \otimes y^{*} \mid\|x\| \leq 1,\left\|y^{*}\right\| \leq 1\right\}$ is norming for $L(X, Y)$.

Proposition 5. Let $\Omega$ be a compact Hausdorff space, $\mu$ a nonnegative finite regular Borel measure on $\Omega,\left(X_{n}\right)_{n \in \mathbb{N}},\left(Y_{n}\right)_{n \in \mathbb{N}}$ two sequences of Banach spaces, $g_{n}: \Omega \rightarrow L\left(X_{n}, Y_{n}\right) \mu$-Bochner integrable functions such that for each $E \in \Sigma_{\Omega}$

$$
\int_{E} g_{n} d \mu \rightarrow 0 \text { in the operator norm. }
$$

Let $U: C(\Omega) \rightarrow c_{0}\left(L\left(X_{n}, Y_{n}\right) \mid n \in \mathbb{N}\right)$ be the operator defined by

$$
U(f)=\left(\int_{\Omega} f(\omega) g_{n}(\omega) d \mu(\omega)\right)_{n \in \mathbb{N}}
$$

Then

(i) $U$ is weakly compact.

(ii) $U$ is compact if and only if $\sup _{\|x\| \leq 1,\left\|y^{*}\right\| \leq 1} \int_{\Omega}\left|\left\langle g_{n}(\omega) x, y^{*}\right\rangle\right| d \mu(\omega) \rightarrow 0$.

(iii) $U$ is absolutely summing if and only if $\int_{\Omega} \sup _{n \in \mathbb{N}}\left\|g_{n}(\omega)\right\| d \mu(\omega)<\infty$.

(iv) $U$ is nuclear if and only if $U$ is absolutely summing and $\left\|g_{n}(\omega)\right\| \rightarrow 0$ $\mu$-a.e. .

Proof. Let $G_{n}: \Sigma_{\Omega} \rightarrow L\left(X_{n}, Y_{n}\right), G_{n}(E)=\int_{E} g_{n} d \mu$ for $E \in \Sigma_{\Omega}$. The hypothesis and Nikodym's boundedness theorem, see [3], gives us that the family 
$\left(\left\langle G_{n} x, y^{*}\right\rangle\right)_{\|x\| \leq 1,\left\|y^{*}\right\| \leq 1, n \in \mathbb{N}}$ is uniformly bounded, i.e., there exists $L>0$ such that

(1)

$$
\int_{\Omega}\left|\left\langle g_{n}(\omega) x, y^{*}\right\rangle\right| d \mu(\omega)=\left|\left\langle G_{n} x, y^{*}\right\rangle\right|(\Omega) \leq L, \forall\|x\| \leq 1,\left\|y^{*}\right\| \leq 1, n \in \mathbb{N} .
$$

From hypothesis we get $\int_{\Omega} f(\omega) g_{n}(\omega) d \mu(\omega) \rightarrow 0$ for each simple function $f$ and from this fact and (1) we deduce $\int_{\Omega} f(\omega) g_{n}(\omega) d \mu(\omega) \rightarrow 0$ for each $f \in B\left(\Sigma_{\Omega}\right)$, so $U$ is well defined. The representing measure of $U$ is

$$
G(E)=\left(\int_{E} g_{n}(\omega) d \mu(\omega)\right)_{n \in \mathbb{N}}, E \in \Sigma_{\Omega}
$$

which, by hypothesis, takes its values in $c_{0}\left(L\left(X_{n}, Y_{n}\right) \mid n \in \mathbb{N}\right)$ and thus, see [3, Chapter VI], $U$ is weakly compact.

By [3, Chapter VI], $U$ is compact if and only if the range of $G$ is relatively norm compact and this by Proposition 1(ii) in [8] is equivalent to $\left\|g_{n}\right\|_{\text {Pettis }} \rightarrow 0$, which by Fact gives (ii).

By [3, Chapter VI], $U$ is absolutely summing if and only if $G$ is of bounded variation, which by Proposition 1(iii) in [8] is equivalent to (iii).

By [3, Chapter VI], $U$ is nuclear if and only if $U$ is absolutely summing and $G$ has a $\mu$-Bochner integrable derivative, and this by Proposition 1(iv) in [8], is equivalent to (iv).

In view of Example 3 in [8] it is a natural question to apply average technique for a triangular matrix of functions. Since by Lemma 4 there is a delineation between averages of first order and averages of order greater or equal than two, we analyze these two situations.

Proposition 6. Let $\Omega$ be a compact Hausdorff space, $\mu$ a nonnegative finite regular Borel measure on $\Omega,\left(X_{n}\right)_{n \in \mathbb{N}}$ a sequence of Banach spaces and $\left(h_{n i}\right)_{1 \leq i \leq n} \subset L_{1}\left(\mu, X_{n}\right)$.

(a) Suppose that

$$
w_{1}\left(\int_{E} h_{n i} d \mu \mid 1 \leq i \leq n ; X_{n}\right) \rightarrow 0 \text { for each } E \in \Sigma_{\Omega} .
$$

Let $U: C(\Omega) \rightarrow c_{0}\left(X_{n} \mid n \in \mathbb{N}\right)$ be the operator defined by

$$
U(f)=\left(\text { Average }\left(\int_{\Omega} f(\omega) h_{n i}(\omega) d \mu(\omega) \mid 1 \leq i \leq n ; X_{n}\right)\right)_{n \in \mathbb{N}} .
$$

Then

(i) $U$ is weakly compact.

(ii) $U$ is compact if and only if

$$
\sup _{\left|\varepsilon_{i}\right| \leq 1}\left\|h_{n 1} \varepsilon_{1}+\cdots+h_{n n} \varepsilon_{n}\right\|_{\text {Pettis }} \rightarrow 0 .
$$


AVERAGES, COMPACT, ABSOLUTELy SUMMing AND NUClEAR OPERATORS 907

(iii) $U$ is absolutely summing if and only if

$$
\int_{\Omega} \sup _{n \in \mathbb{N}} w_{1}\left(h_{n i}(\omega) \mid 1 \leq i \leq n ; X_{n}\right) d \mu(\omega)<\infty .
$$

(iv) $U$ is nuclear if and only if $U$ is absolutely summing and

$$
w_{1}\left(h_{n i}(\omega) \mid 1 \leq i \leq n ; X_{n}\right) \rightarrow 0 \text { for } \mu \text {-a.e. } \omega \in \Omega .
$$

(b) Let $k \geq 2$ be a natural number. Suppose that

$$
b_{n k} w_{2}\left(\int_{E} h_{n i} d \mu \mid 1 \leq i \leq n ; X_{n}\right) \rightarrow 0 \text { for each } E \in \Sigma_{\Omega} .
$$

Let $U: C(\Omega) \rightarrow c_{0}\left(X_{n} \mid n \in \mathbb{N}\right)$ be the operator defined by

$$
U(f)=\left(\text { Average }_{k}\left(\int_{\Omega} f(\omega) h_{n i}(\omega) d \mu(\omega) \mid 1 \leq i \leq n ; X_{n}\right)\right)_{n \in \mathbb{N}} .
$$

Then

(i) $U$ is weakly compact.

(ii) $U$ is compact if and only if

$$
b_{n k} \sup _{\|\xi\|_{2} \leq 1}\left\|h_{n 1} \xi_{1}+\cdots+h_{n n} \xi_{n}\right\|_{\text {Pettis }} \rightarrow 0 .
$$

(iii) $U$ is absolutely summing if and only if

$$
\int_{\Omega} \sup _{n \in \mathbb{N}} b_{n k} w_{2}\left(h_{n i}(\omega) \mid 1 \leq i \leq n ; X_{n}\right) d \mu(\omega)<\infty .
$$

(iv) $U$ is nuclear if and only if $U$ is absolutely summing and

$$
b_{n k} w_{2}\left(h_{n i}(\omega) \mid 1 \leq i \leq n ; X_{n}\right) \rightarrow 0 \text { for } \mu \text {-a.e. } \omega \in \Omega .
$$

Proof. (a) Let $g_{n}: \Omega \rightarrow L\left(X_{n}^{*}, l_{1}^{n}\right)$ be the function defined by

$$
\left(g_{n}(\omega)\right)\left(x^{*}\right)=\left(x^{*}\left(h_{n 1}(\omega)\right), \ldots, x^{*}\left(h_{n n}(\omega)\right)\right) \text { for } \omega \in \Omega, x^{*} \in X_{n}^{*}
$$

i.e., $g_{n}=\sum_{i=1}^{n} h_{n i} \otimes e_{n i}$, and $V_{n}: C(\Omega) \rightarrow L\left(X_{n}^{*}, l_{1}^{n}\right)$

$\left(V_{n}(f)\right)\left(x^{*}\right)=\left(\int_{\Omega} f(\omega) x^{*}\left(h_{n 1}(\omega)\right) d \mu(\omega), \ldots, \int_{\Omega} f(\omega) x^{*}\left(h_{n n}(\omega)\right) d \mu(\omega)\right)$.

Observe that

$$
V_{n}(f)=\int_{\Omega} f(\omega) g_{n}(\omega) d \mu(\omega) .
$$

Further, because $g_{n}$ is obvious Bochner integrable, by Hille's theorem, see [3, Chapter II, Theorem 2.6, p. 47], for each $E \in \Sigma_{\Omega}, x^{*} \in X_{n}^{*}$, we have $\left(\int_{E} g_{n}(\omega) d \mu(\omega)\right)\left(x^{*}\right)=\left(\int_{E} x^{*}\left(h_{n 1}(\omega)\right) d \mu(\omega), \ldots, \int_{E} x^{*}\left(h_{n n}(\omega)\right) d \mu(\omega)\right)$ and thus

$$
\left\|\int_{E} g_{n}(\omega) d \mu(\omega)\right\|_{L\left(X_{n}^{*}, l_{1}^{n}\right)}=w_{1}\left(\int_{E} h_{n i}(\omega) d \mu(\omega) \mid 1 \leq i \leq n ; X_{n}\right)
$$


which, by hypothesis, is convergent to zero. Proposition 5 assures that the operator $V: C(\Omega) \rightarrow c_{0}\left(L\left(X_{n}^{*}, l_{1}^{n}\right) \mid n \in \mathbb{N}\right)$ defined by

$$
V(f)=\left(V_{n}(f)\right)_{n \in \mathbb{N}}
$$

takes its values in $c_{0}\left(L\left(X_{n}^{*}, l_{1}^{n}\right) \mid n \in \mathbb{N}\right)$.

Let $f \in C(\Omega)$. From Lemma 4 we have

$$
\begin{gathered}
\| \text { Average }\left(\int_{\Omega} f(\omega) h_{n i}(\omega) d \mu(\omega) \mid 1 \leq i \leq n ; X_{n}\right) \|_{\infty} \\
\asymp w_{1}\left(\int_{\Omega} f(\omega) h_{n i}(\omega) d \mu(\omega) \mid 1 \leq i \leq n ; X_{n}\right)=\left\|V_{n}(f)\right\|
\end{gathered}
$$

and hence $U$ takes its values in $c_{0}\left(X_{n} \mid n \in \mathbb{N}\right)$ if and only if $V$ takes its values in $c_{0}\left(L\left(X_{n}^{*}, l_{1}^{n}\right) \mid n \in \mathbb{N}\right)$, which, as we already proved, is true.

From (1) we deduce

$$
c\|V(f)\| \leq\|U(f)\| \leq C\|V(f)\|
$$

for some constants $c, C>0$ independent of $f$.

This shows that $U$ is compact (resp. $U$ is absolutely summing) if and only if $V$ is compact (resp. $V$ is absolutely summing) which by Proposition 5 gives (ii) and (iii).

Since (i) and (iv) do not follow from Proposition 5, we argue as follows. The representing measure of $U$ is

$$
G(E)=\left(\text { Average }\left(\int_{E} h_{n i}(\omega) d \mu(\omega) \mid 1 \leq i \leq n ; X_{n}\right)\right)_{n \in \mathbb{N}} \text { for } E \in \Sigma_{\Omega} .
$$

From Lemma 4 and hypothesis, for each $E \in \Sigma_{\Omega}$

$$
\begin{aligned}
& \| \text { Average }\left(\int_{E} h_{n i}(\omega) d \mu(\omega) \mid 1 \leq i \leq n ; X_{n}\right) \|_{\infty} \\
\asymp & w_{1}\left(\int_{E} h_{n i}(\omega) d \mu(\omega) \mid 1 \leq i \leq n ; X_{n}\right) \rightarrow 0,
\end{aligned}
$$

thus $U$ is weakly compact.

By [3, Chapter VI], $U$ is nuclear if and only if $U$ is absolutely summing and $G$ has a $\mu$-Bochner integrable derivative, and this, by Proposition 1(iv) in [8], is equivalent to

$$
\| \text { Average }\left(h_{n i}(\omega) \mid 1 \leq i \leq n ; X_{n}\right) \|_{\infty} \rightarrow 0 \text { for } \mu \text {-a.e. } \omega \in \Omega \text {. }
$$

Then (iv) follows, because by Lemma 4 , for each $\omega \in \Omega$

$$
\| \text { Average }\left(h_{n i}(\omega) \mid 1 \leq i \leq n ; X_{n}\right) \|_{\infty} \asymp w_{1}\left(h_{n i}(\omega) \mid 1 \leq i \leq n ; X_{n}\right) .
$$

(b) As we will see in the sequel the proof of (b) is similar to that of (a). Indeed, in this case, let $g_{n}: \Omega \rightarrow L\left(X_{n}^{*}, l_{2}^{n}\right)$ be the function defined by

$$
\left(g_{n}(\omega)\right)\left(x^{*}\right)=b_{n k}\left(x^{*}\left(h_{n 1}(\omega)\right), \ldots, x^{*}\left(h_{n n}(\omega)\right)\right) \text { for } \omega \in \Omega, x^{*} \in X_{n}^{*},
$$



$V_{n}: C(\Omega) \rightarrow L\left(X_{n}^{*}, l_{2}^{n}\right)$ the operator defined by $\left(V_{n}(f)\right)\left(x^{*}\right)=b_{n k}\left(\int_{\Omega} f(\omega) x^{*}\left(h_{n 1}(\omega)\right) d \mu(\omega), \ldots, \int_{\Omega} f(\omega) x^{*}\left(h_{n n}(\omega)\right) d \mu(\omega)\right)$ and observe that

$$
V_{n}(f)=\int_{\Omega} f(\omega) g_{n}(\omega) d \mu(\omega) .
$$

Further, because $g_{n}$ is Bochner integrable, as in (a) we deduce that for each $E \in \Sigma_{\Omega}$

$$
\left\|\int_{E} g_{n}(\omega) d \mu(\omega)\right\|_{L\left(X_{n}^{*}, l_{2}^{n}\right)}=b_{n k} w_{2}\left(\int_{E} h_{n i}(\omega) d \mu(\omega) \mid 1 \leq i \leq n ; X_{n}\right)
$$

which, by hypothesis, is convergent to zero. Proposition 5 assures that the operator $V: C(\Omega) \rightarrow c_{0}\left(L\left(X_{n}^{*}, l_{2}^{n}\right) \mid n \in \mathbb{N}\right)$ defined by

$$
V(f)=\left(V_{n}(f)\right)_{n \in \mathbb{N}}
$$

takes its values in $c_{0}\left(L\left(X_{n}^{*}, l_{2}^{n}\right) \mid n \in \mathbb{N}\right)$.

Let $f \in C(\Omega)$. From Lemma 4 we have

$$
\begin{gathered}
\| \text { Average }_{k}\left(\int_{\Omega} f(\omega) h_{n i}(\omega) d \mu(\omega) \mid 1 \leq i \leq n ; X_{n}\right) \|_{\infty} \\
\asymp b_{n k} w_{2}\left(\int_{\Omega} f(\omega) h_{n i}(\omega) d \mu(\omega) \mid 1 \leq i \leq n ; X_{n}\right)=\left\|V_{n}(f)\right\|
\end{gathered}
$$

and hence $U$ takes its values in $c_{0}\left(X_{n} \mid n \in \mathbb{N}\right)$ if and only if $V$ takes its values in $c_{0}\left(L\left(X_{n}^{*}, l_{2}^{n}\right) \mid n \in \mathbb{N}\right)$, which is true.

From (2) we deduce

$$
c\|V(f)\| \leq\|U(f)\| \leq C\|V(f)\|
$$

for some constants $c, C>0$ independent of $f$.

This shows that $U$ is compact (resp. $U$ is absolutely summing) if and only if $V$ is compact (resp. $V$ is absolutely summing) and Proposition 5 gives (ii) and (iii).

The representing measure of $U$ is

$$
G(E)=\left(\text { Average }_{k}\left(\int_{E} h_{n i}(\omega) d \mu(\omega) \mid 1 \leq i \leq n ; X_{n}\right)\right)_{n \in \mathbb{N}} \quad \text { for } E \in \Sigma_{\Omega} \text {. }
$$

From Lemma 4 , for each $E \in \Sigma_{\Omega}$

$$
\begin{gathered}
\quad \| \text { Average }_{k}\left(\int_{E} h_{n i}(\omega) d \mu(\omega) \mid 1 \leq i \leq n ; X_{n}\right) \|_{\infty} \\
\asymp b_{n k} w_{2}\left(\int_{E} h_{n i}(\omega) d \mu(\omega) \mid 1 \leq i \leq n ; X_{n}\right) \rightarrow 0
\end{gathered}
$$

by hypothesis, thus $U$ is weakly compact. 
By [3, Chapter VI], $U$ is nuclear if and only if $U$ is absolutely summing and $G$ has a $\mu$-Bochner integrable derivative, and this by Proposition 1(iv) in [8], is equivalent to

$$
\| \text { Average }_{k}\left(h_{n i}(\omega) \mid 1 \leq i \leq n ; X_{n}\right) \|_{\infty} \rightarrow 0 \text { for } \mu \text {-a.e. } \omega \in \Omega .
$$

Then (iv) follows, because by Lemma 4 , for each $\omega \in \Omega$

$$
\| \text { Average }_{k}\left(h_{n i}(\omega) \mid 1 \leq i \leq n ; X_{n}\right) \|_{\infty} \asymp b_{n k} w_{2}\left(h_{n i}(\omega) \mid 1 \leq i \leq n ; X_{n}\right) .
$$

In the next corollary, item (a) is an obvious extension of Example 3 in [8]. In addition to [8], it is natural to study the same problem for averages of order greater or equal than two, i.e., item (b).

Corollary 7. Let $\left(X_{n}\right)_{n \in \mathbb{N}}$ be a sequence of Banach spaces, $\left(x_{n i}\right)_{1 \leq i \leq n} \subset X_{n}$.

(a) Suppose $\sup _{n \in \mathbb{N}} w_{2}\left(x_{n i} \mid 1 \leq i \leq n ; X_{n}\right)<\infty$ and let $U: C[0,1] \rightarrow$ $c_{0}\left(X_{n} \mid n \in \mathbb{N}\right)$ be the operator defined by

$$
U(f)=\left(\text { Average }\left(x_{n i} \int_{0}^{1} f(t) r_{n+i}(t) d t \mid 1 \leq i \leq n ; X_{n}\right)\right)_{n \in \mathbb{N}} .
$$

Then

(i) $U$ is weakly compact.

(ii) $U$ is compact if and only if $w_{2}\left(x_{n i} \mid 1 \leq i \leq n ; X_{n}\right) \rightarrow 0$.

(iii) $U$ is absolutely summing if and only if $\sup _{n \in \mathbb{N}} w_{1}\left(x_{n i} \mid 1 \leq i \leq n ; X_{n}\right)<$ $\infty$.

(iv) $U$ is nuclear if and only if $w_{1}\left(x_{n i} \mid 1 \leq i \leq n ; X_{n}\right) \rightarrow 0$.

(b) Let $k \geq 2$ be a natural number. Suppose $\sup _{n \in \mathbb{N}}\left(b_{n k} \max _{1 \leq i \leq n}\left\|x_{n i}\right\|\right)<\infty$ and let $U: C[0,1] \rightarrow c_{0}\left(X_{n} \mid n \in \mathbb{N}\right)$ be the operator defined by

$$
U(f)=\left(\text { Average }_{k}\left(x_{n i} \int_{0}^{1} f(t) r_{n+i}(t) d t \mid 1 \leq i \leq n ; X_{n}\right)\right)_{n \in \mathbb{N}} .
$$

Then

(i) $U$ is weakly compact.

(ii) $U$ is compact if and only if $b_{n k} \max _{1 \leq i \leq n}\left\|x_{n i}\right\| \rightarrow 0$.

(iii) $U$ is absolutely summing if and only if $\sup _{n \in \mathbb{N}} b_{n k} w_{2}\left(x_{n i} \mid 1 \leq i \leq n ; X_{n}\right)<$ $\infty$.

(iv) $U$ is nuclear if and only if $b_{n k} w_{2}\left(x_{n i} \mid 1 \leq i \leq n ; X_{n}\right) \rightarrow 0$.

Proof. Take $h_{n i}=x_{n i} r_{n+i} \in L_{1}\left(\lambda, X_{n}\right)$ in Proposition 6 .

(a) For each $E \in \mathcal{B}$, by Cauchy-Buniakowski-Schwartz's inequality and hypothesis we have

$$
\begin{aligned}
& w_{1}\left(\int_{E} x_{n i} r_{n+i}(t) d t \mid 1 \leq i \leq n ; X_{n}\right) \\
= & \sup _{\left\|x^{*}\right\| \leq 1}\left(\left|x^{*}\left(x_{n 1}\right)\right|\left|\int_{E} r_{n+1}(t) d t\right|+\cdots+\left|x^{*}\left(x_{n n}\right)\right|\left|\int_{E} r_{2 n}(t) d t\right|\right)
\end{aligned}
$$


AVERAGES, COMPACT, ABSOLUTELY SUMMING AND NUCLEAR OPERATORS 911

$\leq w_{2}\left(x_{n i} \mid 1 \leq i \leq n ; X_{n}\right)\left(\left|\int_{E} r_{n+1}(t) d t\right|^{2}+\cdots+\left|\int_{E} r_{2 n}(t) d t\right|^{2}\right)^{\frac{1}{2}} \rightarrow 0$.

(i) follows from Proposition 6(a)(i).

From the definition of Pettis norm and Khinchin's inequality we get

$$
\sup _{\left|\varepsilon_{i}\right| \leq 1}\left\|x_{n 1} r_{n+1} \varepsilon_{1}+\cdots+x_{n n} r_{2 n} \varepsilon_{n}\right\|_{\text {Pettis }} \asymp w_{2}\left(x_{n i} \mid 1 \leq i \leq n ; X_{n}\right)
$$

and (ii) follows from Proposition 6(a)(ii).

Further for each $t \in[0,1],\left|r_{n}(t)\right|=1$,

$$
w_{1}\left(x_{n i} r_{n+i}(t) \mid 1 \leq i \leq n ; X_{n}\right)=w_{1}\left(x_{n i} \mid 1 \leq i \leq n ; X_{n}\right)
$$

and (iii), (iv) follow from Proposition 6(a)(iii), (iv).

(b) We observe that the hypothesis in Proposition 6(b) are satisfies because, in our hypothesis, for each $E \in \mathcal{B}$

$$
\begin{aligned}
& b_{n k} w_{2}\left(x_{n i} \int_{E} r_{n+i}(t) d t \mid 1 \leq i \leq n ; X_{n}\right) \\
= & b_{n k} \sup _{\left\|x^{*}\right\| \leq 1}\left(\left|x^{*}\left(x_{n 1}\right)\right|^{2}\left|\int_{E} r_{n+1}(t) d t\right|^{2}+\cdots+\left|x^{*}\left(x_{n n}\right)\right|^{2}\left|\int_{E} r_{2 n}(t) d t\right|^{2}\right)^{\frac{1}{2}} \\
\leq & \left(b_{n k} \max _{1 \leq i \leq n}\left\|x_{n i}\right\|\right)\left(\left|\int_{E} r_{n+1}(t) d t\right|^{2}+\cdots+\left|\int_{E} r_{2 n}(t) d t\right|^{2}\right)^{\frac{1}{2}} \rightarrow 0 .
\end{aligned}
$$

(i) follows from Proposition 6(b)(i). From the definition of Pettis norm, Khinchin's inequality and Result we get

$$
\begin{aligned}
& \sup _{\|\xi\|_{2} \leq 1}\left\|x_{n 1} r_{n+1} \xi_{1}+\cdots+x_{n n} r_{2 n} \xi_{n}\right\|_{\text {Pettis }} \\
\asymp & \sup _{\left\|x^{*}\right\| \leq 1} \sup _{\|\xi\|_{2} \leq 1}\left\|\left(\xi_{1} x^{*}\left(x_{n 1}\right), \ldots, \xi_{n} x^{*}\left(x_{n n}\right)\right)\right\|_{2} \\
= & \sup _{\left\|x^{*}\right\| \leq 1}\left\|\left(x^{*}\left(x_{n 1}\right), \ldots, x^{*}\left(x_{n n}\right)\right)\right\|_{\infty} \\
= & \max _{1 \leq i \leq n} \sup _{\left\|x^{*}\right\| \leq 1}\left|x^{*}\left(x_{n i}\right)\right| \\
= & \max _{1 \leq i \leq n}\left\|x_{n i}\right\| .
\end{aligned}
$$

From Proposition 6(b)(ii) we get (ii).

(iii), (iv) follow from Proposition 6(b)(iii), (iv), because for each $t \in[0,1]$

$$
w_{2}\left(x_{n i} r_{n+i}(t) \mid 1 \leq i \leq n ; X_{n}\right)=w_{2}\left(x_{n i} \mid 1 \leq i \leq n ; X_{n}\right) .
$$

Remark. As in the proof of Example 3 in [8], it can be proved that for the operator $U$ defined as in Corollary 7 , either $U$ is absolutely summing, or its representing measure is of everywhere infinite variation, see [4]. 


\section{The examples}

In our examples, in view of Corollary 7, we need the following well-known result. For the sake of completeness we include a short proof.

Proposition 8. (i) Let $X$ be a Banach space, $A \subset B_{X^{*}}$ norming for $X$, $\left(x_{i}\right)_{1 \leq i \leq n} \subset X$ and $1 \leq p<\infty$. Then

$$
w_{p}\left(x_{i} \mid 1 \leq i \leq n ; X\right)=\sup _{x^{*} \in A}\left\|\left(x^{*}\left(x_{1}\right), \ldots, x^{*}\left(x_{n}\right)\right)\right\|_{p} .
$$

(ii) Let $\Omega$ be a compact Hausdorff space, $\left(f_{i}\right)_{1 \leq i \leq n} \subset C(\Omega), f: \Omega \rightarrow \mathbb{K}^{n}$, $f(\omega)=\left(f_{1}(\omega), \ldots, f_{n}(\omega)\right)$ and $1 \leq p<\infty$. Then

$$
w_{p}\left(f_{i} \mid 1 \leq i \leq n ; C(\Omega)\right)=\|f\|_{C\left(\Omega, l_{p}^{n}\right)} .
$$

(iii) Let $X, Y$ be Banach spaces, $U \in L(X, Y), M>0$ such that $\|x\| \leq$ $\|U(x)\| \leq M\|x\|$ for any $x \in X$. Let $\left(x_{i}\right)_{1 \leq i \leq n} \subset X$ and $1 \leq p<\infty$. Then

$$
\begin{aligned}
w_{p}\left(x_{i} \mid 1 \leq i \leq n ; X\right) & \leq w_{p}\left(U\left(x_{i}\right) \mid 1 \leq i \leq n ; Y\right) \\
& \leq M w_{p}\left(x_{i} \mid 1 \leq i \leq n ; X\right) .
\end{aligned}
$$

(iv) Let $1 \leq r<\infty, 1 \leq p \leq \infty, \lambda=\left(\lambda_{1}, \ldots, \lambda_{n}\right) \in \mathbb{K}^{n}$. Then

$$
w_{r}\left(\lambda_{i} e_{n i} \mid 1 \leq i \leq n ; l_{p}^{n}\right)=\left\|M_{\lambda}: l_{r^{*}}^{n} \rightarrow l_{p}^{n}\right\|=\|\lambda\|_{\infty} \text { if } p \geq r^{*},
$$

or $\|\lambda\|_{s}$ if $p<r^{*}$, where $\frac{1}{r}=1-\frac{1}{p}+\frac{1}{s}$.

(v) Let $1 \leq p<\infty, x_{i}=\left(x_{i 1}, \ldots, x_{i n}\right) \in \mathbb{K}^{n}, \beta_{j}=\left(x_{1 j}, \ldots, x_{n j}\right)$ and $\beta=\left(\beta_{1}, \ldots, \beta_{n}\right)$. Then

$$
w_{p}\left(x_{i} \mid 1 \leq i \leq n ; l_{\infty}^{n}\right)=\|\beta\|_{l_{\infty}^{n}\left(l_{p}^{n}\right)} .
$$

(vi) Let $1 \leq p, r<\infty$ and $\lambda=\left(\lambda_{1}, \ldots, \lambda_{n}\right) \in \mathbb{K}^{n}$. Then

$$
w_{r}\left(\lambda_{i} r_{i} \mid 1 \leq i \leq n ; L_{p}[0,1]\right) \asymp\|\lambda\|_{\infty} \text { if } 2 \leq r,
$$

or $\|\lambda\|_{s}$ if $1 \leq r<2$, where $\frac{1}{s}=\frac{1}{r}-\frac{1}{2}$.

(vii) Let $(S, \Sigma, \mu)$ be a measure space, $\left\{E_{1}, \ldots, E_{n}\right\} \subset \Sigma$ pairwise disjoint with $\mu\left(E_{i}\right)<\infty$ for each $1 \leq i \leq n, 1 \leq p, r<\infty$. Then

$$
w_{r}\left(\lambda_{i} \chi_{E_{i}} \mid 1 \leq i \leq n ; L_{p}(\mu)\right)=\max _{1 \leq i \leq n}\left(\left|\lambda_{i}\right|\left[\mu\left(E_{i}\right)\right]^{\frac{1}{p}}\right) \text { if } r^{*} \leq p,
$$

or $\left(\sum_{i=1}^{n}\left|\lambda_{i}\right|^{s}\left[\mu\left(E_{i}\right)\right]^{\frac{s}{p}}\right)^{\frac{1}{s}}$ if $r^{*}>p$, where $\frac{1}{p}=\frac{1}{r^{*}}+\frac{1}{s}$.

(viii) Let $(S, \Sigma, \mu)$ be a measure space, $1 \leq r<\infty,\left(g_{i}\right)_{1 \leq i \leq n} \subset L_{\infty}(\mu)$, $g: S \rightarrow \mathbb{K}^{n}, g(s)=\left(g_{1}(s), \ldots, g_{n}(s)\right)$. Then

$$
w_{r}\left(g_{i} \mid 1 \leq i \leq n ; L_{\infty}(\mu)\right)=\|g\|_{L_{\infty}\left(\mu, l_{r}^{n}\right)} .
$$

(ix) Let $(S, \Sigma, \mu)$ be a finite measure space, $1 \leq r<\infty,\left(g_{i}\right)_{1 \leq i \leq n} \subset L_{1}(\mu)$ such that each $g_{i}$ takes positive values. Then

$$
w_{r}\left(g_{i} \mid 1 \leq i \leq n ; L_{1}(\mu)\right)=\left\|\left(\int_{S} g_{1} d \mu, \ldots, \int_{S} g_{n} d \mu\right)\right\|_{r} .
$$


AVERAGES, COMPACT, ABSOLUTELy SUMMing AND NUCLEAR OPERATORS 913

(x) Let $(S, \Xi, \nu)$ be a finite measure space, $1 \leq r<\infty,\left(g_{i}\right)_{1 \leq i \leq n} \subset L_{1}(\nu)$ and $g: S \rightarrow \mathbb{K}^{n}$ defined by $g(s)=\left(g_{1}(s), \ldots, g_{n}(s)\right)$. Then

$$
w_{1}\left(r_{i} g_{i} \mid 1 \leq i \leq n ; L_{1}(\lambda \otimes \nu)\right) \asymp \int_{S}\|g(s)\|_{2} d \nu(s)
$$

and if $r>1$

$$
w_{r}\left(r_{i} g_{i} \mid 1 \leq i \leq n ; L_{1}(\lambda \otimes \nu)\right) \asymp \sup _{\beta \in l_{r^{*}}^{n},\|\beta\|_{r^{*}} \leq 1} \int_{S}\left\|M_{\beta}(g(s))\right\|_{2} d \nu(s) .
$$

(xi) Let $(S, \Xi, \nu)$ be a finite measure space, $1 \leq r<\infty,\left(E_{i}\right)_{1 \leq i \leq n} \subset \Xi a$ partition of $S,\left(a_{i}\right)_{1 \leq i \leq n} \subset \mathbb{K}$. Then

$$
w_{r}\left(a_{i} r_{i} \chi_{E_{i}} \mid 1 \leq i \leq n ; L_{1}(\lambda \otimes \nu)\right) \asymp\left\|\left(a_{1} \nu\left(E_{1}\right), \ldots, a_{n} \nu\left(E_{n}\right)\right)\right\|_{r} .
$$

Proof. (i) See [6, Lemma 1.1.15, p. 40] where the proof use the equality

$$
w_{p}\left(x_{i} \mid 1 \leq i \leq n ; X\right)=\sup _{\|\lambda\|_{p^{*}} \leq 1}\left\|\lambda_{1} x_{1}+\cdots+\lambda_{n} x_{n}\right\| .
$$

(ii) See [6, Example 1.1.16, p. 40]; it is a particular case of (i), $\left\{\delta_{\omega} \mid \omega \in \Omega\right\}$ being norming for $C(\Omega)$.

(iii) and (iv) follow from hypothesis and (1).

(v) By (1) we have

$$
\begin{aligned}
w_{p}\left(x_{i} \mid 1 \leq i \leq n ; l_{\infty}^{n}\right) & =\sup _{\|\lambda\|_{p^{*}} \leq 1}\left\|\lambda_{1} x_{1}+\cdots+\lambda_{n} x_{n}\right\|_{\infty} \\
& =\sup _{\|\lambda\|_{p^{*}} \leq 1} \max _{1 \leq j \leq n}\left|\lambda_{1} x_{1 j}+\cdots+\lambda_{n} x_{n j}\right| \\
& =\max _{1 \leq j \leq n}\left\|\left(x_{1 j}, \ldots, x_{n j}\right)\right\|_{p}=\|\beta\|_{l_{\infty}^{n}\left(l_{p}^{n}\right)} .
\end{aligned}
$$

(vi) From (1) and Khinchin's inequality

$$
\begin{aligned}
& w_{r}\left(\lambda_{i} r_{i} \mid 1 \leq i \leq n ; L_{p}[0,1]\right)=\sup _{\beta \in l_{r^{*}}^{n},\|\beta\| \leq 1}\left\|\sum_{i=1}^{n} \lambda_{i} \beta_{i} r_{i}\right\|_{L_{p}[0,1]} \\
\asymp & \sup _{\beta \in l_{r^{*}}^{n},\|\beta\| \leq 1}\left(\sum_{i=1}^{n}\left|\lambda_{i} \beta_{i}\right|^{2}\right)^{\frac{1}{2}}=\left\|M_{\lambda}: l_{r^{*}}^{n} \rightarrow l_{2}^{n}\right\| .
\end{aligned}
$$

The assertion follows from well-known formula of the norm of the multiplication operator.

(vii) Again (1) gives

$$
\begin{aligned}
& w_{r}\left(\lambda_{i} \chi_{E_{i}} \mid 1 \leq i \leq n ; L_{p}(\mu)\right) \\
= & \sup _{\beta \in l_{r^{*}}^{n},\|\beta\| \leq 1}\left\|\sum_{i=1}^{n} \lambda_{i} \beta_{i} \chi_{E_{i}}\right\|_{L_{p}(\mu)}
\end{aligned}
$$




$$
=\sup _{\beta \in l_{r^{*}}^{n},\|\beta\| \leq 1}\left(\sum_{i=1}^{n}\left|\lambda_{i} \beta_{i}\right|^{p} \mu\left(E_{i}\right)\right)^{\frac{1}{p}}=\left\|M_{\nu}: l_{r^{*}}^{n} \rightarrow l_{p}^{n}\right\|,
$$

where $\nu=\left(\lambda_{1}\left[\mu\left(E_{1}\right)\right]^{\frac{1}{p}}, \ldots, \lambda_{n}\left[\mu\left(E_{n}\right)\right]^{\frac{1}{p}}\right)$. We use again the norm of the multiplication operator.

(viii) See [6, Example 1.1.17, p. 40].

(ix) By (1) we have

$$
w_{r}\left(g_{i} \mid 1 \leq i \leq n ; L_{1}(\mu)\right)=\sup _{\|\beta\|_{r^{*} \leq 1}} \int_{S}\left|\sum_{i=1}^{n} \beta_{i} g_{i}\right| d \mu .
$$

For each $\|\beta\|_{r^{*}} \leq 1$ we have

$$
\left|\sum_{i=1}^{n} \beta_{i} \int_{S} g_{i} d \mu\right| \leq \int_{S}\left|\sum_{i=1}^{n} \beta_{i} g_{i}\right| d \mu
$$

and from here, by Hölder's inequality

$$
\left\|\left(\int_{S} g_{1} d \mu, \ldots, \int_{S} g_{n} d \mu\right)\right\|_{r} \leq w_{r}\left(g_{i} \mid 1 \leq i \leq n ; L_{1}(\mu)\right) .
$$

For the right inequality, from $\int_{S}\left|\sum_{i=1}^{n} \beta_{i} g_{i}\right| d \mu \leq \sum_{i=1}^{n}\left|\beta_{i}\right| \int_{S}\left|g_{i}\right| d \mu$, Hölder's inequality gives

$$
w_{r}\left(g_{i} \mid 1 \leq i \leq n ; L_{1}(\mu)\right) \leq\left\|\left(\int_{S}\left|g_{1}\right| d \mu, \ldots, \int_{S}\left|g_{n}\right| d \mu\right)\right\|_{r} .
$$

Because each $g_{i}$ takes positive values the statement follows.

(x) By (1)

$$
w_{r}\left(r_{i} g_{i} \mid 1 \leq i \leq n ; L_{1}(\lambda \otimes \nu)\right)=\sup _{\|\beta\|_{r^{*}} \leq 1} \int_{[0,1] \times S}\left|\sum_{i=1}^{n} \beta_{i} r_{i}(t) g_{i}(s)\right| d t d \nu(s) \text {. }
$$

The Fubini theorem gives

$$
\int_{[0,1] \times S}\left|\sum_{i=1}^{n} \beta_{i} r_{i}(t) g_{i}(s)\right| d t d \nu(s)=\int_{S}\left(\int_{0}^{1}\left|\sum_{i=1}^{n} \beta_{i} r_{i}(t) g_{i}(s)\right| d t\right) d \nu(s) \text {. }
$$

Since for each $s \in S$, by Khinchin's inequality,

$$
\int_{0}^{1}\left|\sum_{i=1}^{n} \beta_{i} r_{i}(t) g_{i}(s)\right| d t \asymp \sqrt{\left|\beta_{1}\right|^{2}\left|g_{1}(s)\right|^{2}+\cdots+\left|\beta_{n}\right|^{2}\left|g_{n}(s)\right|^{2}}
$$

by integration we obtain

$$
w_{r}\left(r_{i} g_{i} \mid 1 \leq i \leq n ; L_{1}(\lambda \otimes \nu)\right) \asymp \sup _{\|\beta\|_{r^{*}} \leq 1} \int_{S}\left\|M_{\beta}(g(s))\right\|_{2} d \nu(s) .
$$

In case $r=1$, we have $\sup _{\|\beta\|_{\infty} \leq 1} \int_{S}\left\|M_{\beta}(g(s))\right\|_{2} d \nu(s)=\int_{S}\|g(s)\|_{2} d \nu(s)$ and the statement follows. 
(xi) is a particular case of (x). In this situation $g: S \rightarrow \mathbb{K}^{n}$ is defined by $g(s)=\left(a_{1} \chi_{E_{1}}(s), \ldots, a_{n} \chi_{E_{n}}(s)\right)$. In case $r=1$, since $\left(E_{i}\right)_{1<i<n}$ is a partition of $S,\|g(s)\|_{2}=\left|a_{1}\right| \chi_{E_{1}}(s)+\cdots+\left|a_{n}\right| \chi_{E_{n}}(s)$ and the statement follows. In case $r>1$, for each $\beta \in l_{r^{*}}^{n}$,

$$
\left\|M_{\beta}(g(s))\right\|_{2}=\left|a_{1}\right|\left|\beta_{1}\right| \chi_{E_{1}}(s)+\cdots+\left|a_{n}\right|\left|\beta_{n}\right| \chi_{E_{n}}(s)
$$

and thus by $(\mathrm{x})$

$$
\begin{aligned}
& w_{r}\left(a_{i} r_{i} \chi_{E_{i}} \mid 1 \leq i \leq n ; L_{1}(\lambda \otimes \nu)\right) \\
\asymp & \sup _{\beta \in l_{r^{*}}^{n},\|\beta\|_{r^{*}} \leq 1}\left(\sum_{i=1}^{n}\left|a_{i}\right|\left|\beta_{i}\right| \nu\left(E_{i}\right)\right)=\left\|\left(a_{1} \nu\left(E_{1}\right), \ldots, a_{n} \nu\left(E_{n}\right)\right)\right\|_{r} .
\end{aligned}
$$

In the next example item (a) is a natural extension of Example 3 in [8]; for $\Omega_{n}=\{1\}$, a singleton, $C\left(\Omega_{n}\right)=\mathbb{K}$. Further, item (b) is a natural completion of the same example. The proof follows from Corollary 7 and Proposition 8(ii).

Example 9. Let $\left(\Omega_{n}\right)_{n \in \mathbb{N}}$ be a sequence of compact Hausdorff spaces,

$$
\left(\varphi_{n i}\right)_{1 \leq i \leq n} \subset C\left(\Omega_{n}\right), \varphi_{n}: \Omega_{n} \rightarrow \mathbb{K}^{n}, \varphi_{n}(\omega)=\left(\varphi_{n 1}(\omega), \ldots, \varphi_{n n}(\omega)\right) \text {. }
$$

(a) Denote $\varphi=\left(\varphi_{n}\right)_{n \in \mathbb{N}}$ and suppose that $\varphi \in l_{\infty}\left(C\left(\Omega_{n}, l_{2}^{n}\right) \mid n \in \mathbb{N}\right)$. Let $U: C[0,1] \rightarrow c_{0}\left(C\left(\Omega_{n}\right) \mid n \in \mathbb{N}\right)$ be the operator defined by

$$
U(f)=\left(\text { Average }\left(\varphi_{n i} \int_{0}^{1} f(t) r_{n+i}(t) d t \mid 1 \leq i \leq n ; C\left(\Omega_{n}\right)\right)\right)_{n \in \mathbb{N}} .
$$

Then

(i) $U$ is weakly compact.

(ii) $U$ is compact if and only if $\varphi \in c_{0}\left(C\left(\Omega_{n}, l_{2}^{n}\right) \mid n \in \mathbb{N}\right)$.

(iii) $U$ is absolutely summing if and only if $\varphi \in l_{\infty}\left(C\left(\Omega_{n}, l_{1}^{n}\right) \mid n \in \mathbb{N}\right)$.

(iv) $U$ is nuclear if and only if $\varphi \in c_{0}\left(C\left(\Omega_{n}, l_{1}^{n}\right) \mid n \in \mathbb{N}\right)$.

(aa) Let $k \geq 2$ be a natural number. Denote $\varphi_{\bmod }=\left(b_{n k} \varphi_{n}\right)_{n \in \mathbb{N}}$ and suppose that $\varphi_{\text {mod }} \in l_{\infty}\left(C\left(\Omega_{n}, l_{\infty}^{n}\right) \mid n \in \mathbb{N}\right)$. Let $U: C[0,1] \rightarrow c_{0}\left(C\left(\Omega_{n}\right) \mid n \in \mathbb{N}\right)$ be the operator defined by

$$
U(f)=\left(\text { Average }_{k}\left(\varphi_{n i} \int_{0}^{1} f(t) r_{n+i}(t) d t \mid 1 \leq i \leq n ; C\left(\Omega_{n}\right)\right)\right)_{n \in \mathbb{N}} .
$$

Then

(i) $U$ is weakly compact.

(ii) $U$ is compact if and only if $\varphi_{\bmod } \in c_{0}\left(C\left(\Omega_{n}, l_{\infty}^{n}\right) \mid n \in \mathbb{N}\right)$.

(iii) $U$ is absolutely summing if and only if $\varphi_{\bmod } \in l_{\infty}\left(C\left(\Omega_{n}, l_{2}^{n}\right) \mid n \in \mathbb{N}\right)$.

(iv) $U$ is nuclear if and only if $\varphi_{\bmod } \in c_{0}\left(C\left(\Omega_{n}, l_{2}^{n}\right) \mid n \in \mathbb{N}\right)$.

To avoid repetitions in Examples 10, 13-16, if $\left(\alpha_{n i}\right)_{1 \leq i \leq n, n \in \mathbb{N}}$ is a triangular matrix of scalars, which in the statement of these examples will be written as $\left(\alpha_{n i}\right)_{i, n}$, we denote $\alpha_{n}=\left(\alpha_{n 1}, \ldots, \alpha_{n n}\right), \alpha=\left(\alpha_{n}\right)_{n \in \mathbb{N}}$ and $\alpha_{\bmod }=\left(b_{n k} \alpha_{n}\right)_{n \in \mathbb{N}}$ for a natural number $k \geq 2$. 
Also, in Examples 13(c), 14(c), 15(cc), if $\left(\alpha_{n i j}\right)_{1 \leq i \leq n, 1 \leq j \leq n, n \in \mathbb{N}} \subset \mathbb{K}$, which in the statement of these examples will be written as $\left(\alpha_{n i j}\right)_{i, j, n}$, we denote $\beta_{n j}=\left(\alpha_{n 1 j}, \ldots, \alpha_{n n j}\right), \beta_{n}=\left(\beta_{n 1}, \ldots, \beta_{n n}\right), \beta=\left(\beta_{n}\right)_{n \in \mathbb{N}}$ and $\beta_{\bmod }=$ $\left(b_{n k} \beta_{n}\right)_{n \in \mathbb{N}}$ for a natural number $k \geq 2$.

The proof of the next example in case $1 \leq p<\infty$ (resp. $p=\infty$ ) follows from Corollary 7 and Proposition 8(vi) (resp. (viii)).

Example 10. (a) Let $1 \leq p<\infty,\left(\alpha_{n i}\right)_{i, n}$ be such that $\alpha \in l_{\infty}\left(l_{\infty}^{n} \mid n \in \mathbb{N}\right)$ and $U: C[0,1] \rightarrow c_{0}\left(L_{p}[0,1]\right)$

$$
U(f)=\left(\text { Average }\left(\alpha_{n i} r_{i} \int_{0}^{1} f(t) r_{n+i}(t) d t \mid 1 \leq i \leq n ; L_{p}[0,1]\right)\right)_{n \in \mathbb{N}} .
$$

Then

(i) $U$ is weakly compact.

(ii) $U$ is compact if and only if $\alpha \in c_{0}\left(l_{\infty}^{n} \mid n \in \mathbb{N}\right)$.

(iii) $U$ is absolutely summing if and only if $\alpha \in l_{\infty}\left(l_{2}^{n} \mid n \in \mathbb{N}\right)$.

(iv) $U$ is nuclear if and only if $\alpha \in c_{0}\left(l_{2}^{n} \mid n \in \mathbb{N}\right)$.

(aa) Let $k \geq 2$ be a natural number, $1 \leq p<\infty,\left(\alpha_{n i}\right)_{i, n}$ such that $\alpha_{\text {mod }} \in$ $l_{\infty}\left(l_{\infty}^{n} \mid n \in \mathbb{N}\right)$ and $U: C[0,1] \rightarrow c_{0}\left(L_{p}[0,1]\right)$

$$
U(f)=\left(\text { Average }_{k}\left(\alpha_{n i} r_{i} \int_{0}^{1} f(t) r_{n+i}(t) d t \mid 1 \leq i \leq n ; L_{p}[0,1]\right)\right)_{n \in \mathbb{N}} .
$$

Then $U$ is absolutely summing; $U$ is compact if and only if $U$ is nuclear if and only if $\alpha_{\bmod } \in c_{0}\left(l_{\infty}^{n} \mid n \in \mathbb{N}\right)$.

(b) Let $\left(\alpha_{n i}\right)_{i, n}$ be such that $\alpha \in l_{\infty}\left(l_{2}^{n} \mid n \in \mathbb{N}\right)$ and $U: C[0,1] \rightarrow c_{0}\left(L_{\infty}[0,1]\right)$ the operator defined by

$$
U(f)=\left(\text { Average }\left(\alpha_{n i} r_{i} \int_{0}^{1} f(t) r_{n+i}(t) d t \mid 1 \leq i \leq n ; L_{\infty}[0,1]\right)\right)_{n \in \mathbb{N}} .
$$

Then

(i) $U$ is weakly compact.

(ii) $U$ is compact if and only if $\alpha \in c_{0}\left(l_{2}^{n} \mid n \in \mathbb{N}\right)$.

(iii) $U$ is absolutely summing if and only if $\alpha \in l_{\infty}\left(l_{1}^{n} \mid n \in \mathbb{N}\right)$.

(iv) $U$ is nuclear if and only if $\alpha \in c_{0}\left(l_{1}^{n} \mid n \in \mathbb{N}\right)$.

(bb) Let $k \geq 2$ be a natural number, $\left(\alpha_{n i}\right)_{i, n}$ such that $\alpha_{\bmod } \in l_{\infty}\left(l_{\infty}^{n} \mid n \in \mathbb{N}\right)$ and $U: C[0,1] \rightarrow c_{0}\left(L_{\infty}[0,1]\right)$

$$
U(f)=\left(\text { Average }_{k}\left(\alpha_{n i} r_{i} \int_{0}^{1} f(t) r_{n+i}(t) d t \mid 1 \leq i \leq n ; L_{\infty}[0,1]\right)\right)_{n \in \mathbb{N}} .
$$

Then

(i) $U$ is weakly compact.

(ii) $U$ is compact if and only if $\alpha_{\bmod } \in c_{0}\left(l_{\infty}^{n} \mid n \in \mathbb{N}\right)$.

(iii) $U$ is absolutely summing if and only if $\alpha_{\bmod } \in l_{\infty}\left(l_{2}^{n} \mid n \in \mathbb{N}\right)$.

(iv) $U$ is nuclear if and only if $\alpha_{\bmod } \in c_{0}\left(l_{2}^{n} \mid n \in \mathbb{N}\right)$. 
The next example is a natural extension of Example 10(b), (bb). The proof follows from Corollary 7 and Proposition 8(viii). We remark that in the next example, if:

1) $S_{n}=[0,1], \mu_{n}=\lambda$ and all $\left(g_{n i}\right)_{1 \leq i \leq n, n \in \mathbb{N}} \subset L_{\infty}\left(\mu_{n}\right)$ are continuous, then we must replace $L_{\infty}\left(\mu_{n}, \cdot\right)$ with $C([0,1], \cdot)$.

2) $S_{n}=[0,1], \mu_{n}=\lambda$ and $g_{n i}=\alpha_{n i} r_{i}$ we get Example $10(\mathrm{~b}),(\mathrm{bb})$.

Example 11. Let $\left(S_{n}, \Sigma_{n}, \mu_{n}\right)_{n \in \mathbb{N}}$ be a sequence of finite measure spaces,

$$
\left(g_{n i}\right)_{1 \leq i \leq n, n \in \mathbb{N}} \subset L_{\infty}\left(\mu_{n}\right), g_{n}: \Omega_{n} \rightarrow \mathbb{K}^{n}, g_{n}=\left(g_{n 1}, \ldots, g_{n n}\right) .
$$

(a) Denote $g=\left(g_{n}\right)_{n \in \mathbb{N}}$ and suppose that $g \in l_{\infty}\left(L_{\infty}\left(\mu_{n}, l_{2}^{n}\right) \mid n \in \mathbb{N}\right)$.

Let $U: C[0,1] \rightarrow c_{0}\left(L_{\infty}\left(\mu_{n}\right) \mid n \in \mathbb{N}\right)$ be the operator defined by

$$
U(f)=\left(\text { Average }\left(g_{n i} \int_{0}^{1} f(t) r_{n+i}(t) d t \mid 1 \leq i \leq n ; L_{\infty}\left(\mu_{n}\right)\right)\right)_{n \in \mathbb{N}} .
$$

Then

(i) $U$ is weakly compact.

(ii) $U$ is compact if and only if $g \in c_{0}\left(L_{\infty}\left(\mu_{n}, l_{2}^{n}\right) \mid n \in \mathbb{N}\right)$.

(iii) $U$ is absolutely summing if and only if $g \in l_{\infty}\left(L_{\infty}\left(\mu_{n}, l_{1}^{n}\right) \mid n \in \mathbb{N}\right)$.

(iv) $U$ is nuclear if and only if $g \in c_{0}\left(L_{\infty}\left(\mu_{n}, l_{1}^{n}\right) \mid n \in \mathbb{N}\right)$.

(aa) Let $k \geq 2$ be a natural number and denote $g_{\text {mod }}=\left(b_{n k} g_{n}\right)_{n \in \mathbb{N}}$. Suppose that $g_{\text {mod }} \in l_{\infty}\left(L_{\infty}\left(\mu_{n}, l_{\infty}^{n}\right) \mid n \in \mathbb{N}\right)$ and let $U: C[0,1] \rightarrow c_{0}\left(L_{\infty}\left(\mu_{n}\right) \mid n \in \mathbb{N}\right)$ be the operator defined by

$$
U(f)=\left(\text { Average }_{k}\left(g_{n i} \int_{0}^{1} f(t) r_{n+i}(t) d t \mid 1 \leq i \leq n\right) ; L_{\infty}\left(\mu_{n}\right)\right)_{n \in \mathbb{N}} .
$$

Then

(i) $U$ is weakly compact.

(ii) $U$ is compact if and only if $g_{\bmod } \in c_{0}\left(L_{\infty}\left(\mu_{n}, l_{\infty}^{n}\right) \mid n \in \mathbb{N}\right)$.

(iii) $U$ is absolutely summing if and only if $g_{\bmod } \in l_{\infty}\left(L_{\infty}\left(\mu_{n}, l_{2}^{n}\right) \mid n \in \mathbb{N}\right)$.

(iv) $U$ is nuclear if and only if $g_{\text {mod }} \in c_{0}\left(L_{\infty}\left(\mu_{n}, l_{2}^{n}\right) \mid n \in \mathbb{N}\right)$.

The proof of the next example follows from Corollary 7 and Proposition 8(ix).

Example 12. Let $\left(S_{n}, \Sigma_{n}, \mu_{n}\right)_{n \in \mathbb{N}}$ be a sequence of finite measure spaces,

$$
\left(g_{n i}\right)_{1 \leq i \leq n, n \in \mathbb{N}} \subset L_{1}\left(\mu_{n}\right) .
$$

(a) Denote $\beta_{n}=\left(\int_{S} g_{n 1} d \mu_{n}, \ldots, \int_{S} g_{n n} d \mu_{n}\right), \beta=\left(\beta_{n}\right)_{n \in \mathbb{N}}$, suppose that each $g_{n i}$ takes positive values and $\beta \in l_{\infty}\left(l_{2}^{n} \mid n \in \mathbb{N}\right)$.

Let $U: C[0,1] \rightarrow c_{0}\left(L_{1}\left(\mu_{n}\right) \mid n \in \mathbb{N}\right)$ be the operator defined by

$$
U(f)=\left(\text { Average }\left(g_{n i} \int_{0}^{1} f(t) r_{n+i}(t) d t \mid 1 \leq i \leq n\right) ; L_{1}\left(\mu_{n}\right)\right)_{n \in \mathbb{N}} .
$$

Then

(i) $U$ is weakly compact.

(ii) $U$ is compact if and only if $\beta \in c_{0}\left(l_{2}^{n} \mid n \in \mathbb{N}\right)$. 
(iii) $U$ is absolutely summing if and only if $\beta \in l_{\infty}\left(l_{1}^{n} \mid n \in \mathbb{N}\right)$.

(iv) $U$ is nuclear if and only if $\beta \in c_{0}\left(l_{1}^{n} \mid n \in \mathbb{N}\right)$.

(aa) Let $k \geq 2$ be a natural number. Denote

$$
\beta_{n}=\left(\int_{S}\left|g_{n 1}\right| d \mu_{n}, \ldots, \int_{S}\left|g_{n n}\right| d \mu_{n}\right),
$$

$\beta_{\text {mod }}=\left(b_{n k} \beta_{n}\right)_{n \in \mathbb{N}}$ and suppose that $\beta_{\bmod } \in l_{\infty}\left(l_{\infty}^{n} \mid n \in \mathbb{N}\right)$.

Let $U: C[0,1] \rightarrow c_{0}\left(L_{1}\left(\mu_{n}\right) \mid n \in \mathbb{N}\right)$ be the operator defined by

$$
U(f)=\left(\text { Average }_{k}\left(g_{n i} \int_{0}^{1} f(t) r_{n+i}(t) d t \mid 1 \leq i \leq n\right) ; L_{1}\left(\mu_{n}\right)\right)_{n \in \mathbb{N}} .
$$

Then

(i) $U$ is weakly compact.

(ii) $U$ is compact if and only if $\beta_{\text {mod }} \in c_{0}\left(l_{\infty}^{n} \mid n \in \mathbb{N}\right)$.

(iii) If, in addition, each $g_{n i}$ takes positive values, $U$ is absolutely summing if and only if $\beta_{\text {mod }} \in l_{\infty}\left(l_{2}^{n} \mid n \in \mathbb{N}\right)$.

(iv) If, in addition, each $g_{n i}$ takes positive values, $U$ is nuclear if and only if $\beta_{\text {mod }} \in c_{0}\left(l_{2}^{n} \mid n \in \mathbb{N}\right)$.

In the rest of the paper, if $1 \leq p<2$ define $r$ by $\frac{1}{p}=\frac{1}{2}+\frac{1}{r}$. The proof of the next example follows from Corollary 7 and Proposition $8(\mathrm{iv})$ and (v).

Example 13. (a) Let $\left(\alpha_{n i}\right)_{i, n}$ be such that $\alpha \in l_{\infty}\left(l_{r}^{n} \mid n \in \mathbb{N}\right)$ if $1 \leq p<2$, or $\alpha \in l_{\infty}\left(l_{\infty}^{n} \mid n \in \mathbb{N}\right)$ if $2 \leq p$ and $U: C[0,1] \rightarrow c_{0}\left(l_{p}^{n} \mid n \in \mathbb{N}\right)$

$$
U(f)=\left(\text { Average }\left(\alpha_{n i} e_{n i} \int_{0}^{1} f(t) r_{n+i}(t) d t \mid 1 \leq i \leq n ; l_{p}^{n}\right)\right)_{n \in \mathbb{N}} .
$$

Then

(i) $U$ is weakly compact.

(ii) $U$ is compact if and only if $\alpha \in c_{0}\left(l_{r}^{n} \mid n \in \mathbb{N}\right)$ for $1 \leq p<2$, or $\alpha \in$ $c_{0}\left(l_{\infty}^{n} \mid n \in \mathbb{N}\right)$ for $2 \leq p$.

(iii) $U$ is absolutely summing if and only if $\alpha \in l_{\infty}\left(l_{p}^{n} \mid n \in \mathbb{N}\right)$.

(iv) $U$ is nuclear if and only if $\alpha \in c_{0}\left(l_{p}^{n} \mid n \in \mathbb{N}\right)$.

(b) Let $\left(\alpha_{n i}\right)_{i, n}$ be such that $\alpha \in l_{\infty}\left(l_{\infty}^{n} \mid n \in \mathbb{N}\right)$ and $U: C[0,1] \rightarrow$ $c_{0}\left(l_{\infty}^{n} \mid n \in \mathbb{N}\right)$ the operator defined by

$$
U(f)=\left(\text { Average }\left(\alpha_{n i} e_{n i} \int_{0}^{1} f(t) r_{n+i}(t) d t \mid 1 \leq i \leq n ; l_{\infty}^{n}\right)\right)_{n \in \mathbb{N}} .
$$

Then $U$ is absolutely summing; $U$ is compact if and only if $U$ is nuclear if and only if $\alpha \in c_{0}\left(l_{\infty}^{n} \mid n \in \mathbb{N}\right)$.

(c) Let $\left(\alpha_{n i j}\right)_{i, j, n}$ be such that $\beta \in l_{\infty}\left(l_{\infty}^{n}\left(l_{2}^{n}\right) \mid n \in \mathbb{N}\right)$ and $U: C[0,1] \rightarrow$ $c_{0}\left(l_{\infty}^{n} \mid n \in \mathbb{N}\right)$ the operator defined by

$U(f)=\left(\text { Average }\left(\left(\alpha_{n i 1} e_{n 1}+\cdots+\alpha_{n i n} e_{n n}\right) \int_{0}^{1} f(t) r_{n+i}(t) d t \mid 1 \leq i \leq n ; l_{\infty}^{n}\right)\right)_{n \in \mathbb{N}}$. 
Then

(i) $U$ is weakly compact.

(ii) $U$ is compact if and only if $\beta \in c_{0}\left(l_{\infty}^{n}\left(l_{2}^{n}\right) \mid n \in \mathbb{N}\right)$.

(iii) $U$ is absolutely summing if and only if $\beta \in l_{\infty}\left(l_{\infty}^{n}\left(l_{1}^{n}\right) \mid n \in \mathbb{N}\right)$.

(iv) $U$ is nuclear if and only if $\beta \in c_{0}\left(l_{\infty}^{n}\left(l_{1}^{n}\right) \mid n \in \mathbb{N}\right)$.

The next example is a natural completion of Example 13. The proof follows from Corollary 7 and Proposition 8(iv) and (v).

Example 14. (a) Let $k \geq 2$ be a natural number, $1 \leq p<\infty,\left(\alpha_{n i}\right)_{i, n}$ such that $\alpha_{\bmod } \in l_{\infty}\left(l_{\infty}^{n} \mid n \in \mathbb{N}\right)$ and $U: C[0,1] \rightarrow c_{0}\left(l_{p}^{n} \mid n \in \mathbb{N}\right)$

$$
U(f)=\left(\text { Average }_{k}\left(\alpha_{n i} e_{n i} \int_{0}^{1} f(t) r_{n+i}(t) d t \mid 1 \leq i \leq n ; l_{p}^{n}\right)\right)_{n \in \mathbb{N}} .
$$

Then

(i) $U$ is weakly compact.

(ii) $U$ is compact if and only if $\alpha_{\text {mod }} \in c_{0}\left(l_{\infty}^{n} \mid n \in \mathbb{N}\right)$.

(iii) for $p \geq 2, U$ is absolutely summing; for $1 \leq p<2, U$ is absolutely summing if and only if $\alpha_{\bmod } \in l_{\infty}\left(l_{r}^{n} \mid n \in \mathbb{N}\right)$.

(iv) for $p \geq 2, U$ is nuclear if and only if $U$ is compact if and only if $\alpha_{\bmod } \in$ $c_{0}\left(l_{\infty}^{n} \mid n \in \mathbb{N}\right)$; for $1 \leq p<2, U$ is nuclear if and only if $\alpha_{\bmod } \in c_{0}\left(l_{r}^{n} \mid n \in \mathbb{N}\right)$.

(b) Let $k \geq 2$ be a natural number, $\left(\alpha_{n i}\right)_{i, n}$ such that $\alpha_{\bmod } \in l_{\infty}\left(l_{\infty}^{n} \mid n \in \mathbb{N}\right)$ and $U: C[0,1] \rightarrow c_{0}\left(l_{\infty}^{n} \mid n \in \mathbb{N}\right)$

$$
U(f)=\left(\text { Average }_{k}\left(\alpha_{n i} e_{n i} \int_{0}^{1} f(t) r_{n+i}(t) d t \mid 1 \leq i \leq n ; l_{\infty}^{n}\right)\right)_{n \in \mathbb{N}}
$$

Then $U$ is absolutely summing; $U$ is compact if and only if $U$ is nuclear if and only if $\alpha_{\text {mod }} \in c_{0}\left(l_{\infty}^{n} \mid n \in \mathbb{N}\right)$.

(c) Let $k \geq 2$ be a natural number, $\left(\alpha_{n i j}\right)_{i, j, n}$ such that $\beta_{\bmod } \in l_{\infty}\left(l_{\infty}^{n}\left(l_{\infty}^{n}\right) \mid\right.$ $n \in \mathbb{N})$ and $U: C[0,1] \rightarrow c_{0}\left(l_{\infty}^{n} \mid n \in \mathbb{N}\right)$

$U(f)=\left(\text { Average }_{k}\left(\left(\alpha_{n i 1} e_{n 1}+\cdots+\alpha_{n i n} e_{n n}\right) \int_{0}^{1} f(t) r_{n+i}(t) d t \mid 1 \leq i \leq n ; l_{\infty}^{n}\right)\right)_{n \in \mathbb{N}}$.

Then

(i) $U$ is weakly compact.

(ii) $U$ is compact if and only if $\beta_{\bmod } \in c_{0}\left(l_{\infty}^{n}\left(l_{\infty}^{n}\right) \mid n \in \mathbb{N}\right)$.

(iii) $U$ is absolutely summing if and only if $\beta_{\bmod } \in l_{\infty}\left(l_{\infty}^{n}\left(l_{2}^{n}\right) \mid n \in \mathbb{N}\right)$.

(iv) $U$ is nuclear if and only if $\beta_{\bmod } \in c_{0}\left(l_{\infty}^{n}\left(l_{2}^{n}\right) \mid n \in \mathbb{N}\right)$.

The Examples 13 and 14 can be extended to a more general situation. For this we recall, that if $1 \leq p \leq \infty$, a Banach space $X$ contains $l_{p}^{n}$ 's uniformly if and only if there exists $\lambda \geq 1$ such that for each $n \in \mathbb{N}$ there exists a bounded linear operator $J: l_{p}^{n} \rightarrow X$ such that

$$
\|\xi\|_{p} \leq\|J(\xi)\|_{X} \leq \lambda\|\xi\|_{p}, \forall \xi \in l_{p}^{n} .
$$


A deep result of Krivine's, see [7, p. 233], asserts that a Banach space $X$ contains $l_{p}^{n}$ 's uniformly if and only if for all $\lambda>1$, all $n \in \mathbb{N}$ there exists a bounded linear operator $J: l_{p}^{n} \rightarrow X$ such that

$$
\|\xi\|_{p} \leq\|J(\xi)\|_{X} \leq \lambda\|\xi\|_{p}, \forall \xi \in l_{p}^{n} .
$$

For example, from Khinchin's inequality it follows that for each $1 \leq p<\infty$, $L_{p}[0,1]$ contains $l_{2}^{n}$ 's uniformly, thus Example $10(\mathrm{a}),($ aa) is a particular case of next example.

From [1, Exercise 8.18(a), p. 107] it follows that $L_{\infty}[0,1]$ contains $l_{1}^{n}$ 's uniformly, thus Example 10(b), (bb) is also a particular case of next example.

In the statement of the next example we will use the operator $J$ which occur in $(*)$.

Example 15. (a) Let $1 \leq p<\infty, X$ be a Banach space which contains $l_{p}^{n}$ 's uniformly, $\left(\alpha_{n i}\right)_{i, n}$ such that $\alpha \in l_{\infty}\left(l_{r}^{n} \mid n \in \mathbb{N}\right)$ if $1 \leq p<2$, or $\alpha \in$ $l_{\infty}\left(l_{\infty}^{n} \mid n \in \mathbb{N}\right)$ if $2 \leq p$. Let $U_{\alpha}: C[0,1] \rightarrow c_{0}(X)$ be the operator defined by

$$
U_{\alpha}(f)=\left(\text { Average }\left(\alpha_{n i} J\left(e_{n i}\right) \int_{0}^{1} f(t) r_{n+i}(t) d t\right) \mid 1 \leq i \leq n\right)_{n \in \mathbb{N}} .
$$

Then

(i) $U_{\alpha}$ is weakly compact.

(ii) $U_{\alpha}$ is compact if and only if $\alpha \in c_{0}\left(l_{r}^{n} \mid n \in \mathbb{N}\right)$ if $1 \leq p<2$, or $\alpha \in$ $c_{0}\left(l_{\infty}^{n} \mid n \in \mathbb{N}\right)$ if $2 \leq p$.

(iii) $U_{\alpha}$ is absolutely summing if and only if $\alpha \in l_{\infty}\left(l_{p}^{n} \mid n \in \mathbb{N}\right)$.

(iv) $U_{\alpha}$ is nuclear if and only if $\alpha \in c_{0}\left(l_{p}^{n} \mid n \in \mathbb{N}\right)$.

(b) Let $X$ be a Banach space which contains $l_{\infty}^{n}$ 's uniformly, $\left(\alpha_{n i}\right)_{i, n}$ such that $\alpha \in l_{\infty}\left(l_{\infty}^{n} \mid n \in \mathbb{N}\right)$. Let $U_{\alpha}: C[0,1] \rightarrow c_{0}(X)$ be the operator defined by

$$
U_{\alpha}(f)=\left(\text { Average }\left(\alpha_{n i} J\left(e_{n i}\right) \int_{0}^{1} f(t) r_{n+i}(t) d t\right) \mid 1 \leq i \leq n\right)_{n \in \mathbb{N}} .
$$

Then

(i) $U_{\alpha}$ is absolutely summing.

(ii) $U_{\alpha}$ is compact if and only if $U_{\alpha}$ is nuclear if and only if $\alpha \in c_{0}\left(l_{\infty}^{n} \mid n \in \mathbb{N}\right)$.

(c) Let $X$ be a Banach space which contains $l_{\infty}^{n}$ 's uniformly, $\left(\alpha_{n i j}\right)_{i, j, n}$ such that $\beta \in l_{\infty}\left(l_{\infty}^{n}\left(l_{2}^{n}\right) \mid n \in \mathbb{N}\right)$ and for each natural number $n$ denote

$$
x_{n i}=\alpha_{n i 1} J\left(e_{n 1}\right)+\alpha_{n i 2} J\left(e_{n 2}\right)+\cdots+\alpha_{n i n} J\left(e_{n n}\right) .
$$

Let $U_{\beta}: C[0,1] \rightarrow c_{0}(X)$ be the operator defined by

$$
\left.U_{\beta}(f)=\left(\text { Average }\left(x_{n i} \int_{0}^{1} f(t) r_{n+i}(t) d t\right) \mid 1 \leq i \leq n\right)\right)_{n \in \mathbb{N}} .
$$

Then

(i) $U_{\beta}$ is weakly compact.

(ii) $U_{\beta}$ is compact if and only if $\beta \in c_{0}\left(l_{\infty}^{n}\left(l_{2}^{n}\right) \mid n \in \mathbb{N}\right)$.

(iii) $U_{\beta}$ is absolutely summing if and only if $\beta \in l_{\infty}\left(l_{\infty}^{n}\left(l_{1}^{n}\right) \mid n \in \mathbb{N}\right)$. 
(iv) $U_{\beta}$ is nuclear if and only if $\beta \in c_{0}\left(l_{\infty}^{n}\left(l_{1}^{n}\right) \mid n \in \mathbb{N}\right)$.

(aa) Let $k \geq 2$ be a natural number, $1 \leq p<\infty, X$ a Banach space which contains $l_{p}^{n}$ 's uniformly, $\left(\alpha_{n i}\right)_{i, n}$ such that $\alpha_{\bmod } \in l_{\infty}\left(l_{\infty}^{n} \mid n \in \mathbb{N}\right)$.

Let $U_{\alpha}: C[0,1] \rightarrow c_{0}(X)$ be the operator defined by

$$
U_{\alpha}(f)=\left(\text { Average }_{k}\left(\alpha_{n i} J\left(e_{n i}\right) \int_{0}^{1} f(t) r_{n+i}(t) d t\right) \mid 1 \leq i \leq n\right)_{n \in \mathbb{N}} .
$$

Then

(i) $U_{\alpha}$ is weakly compact.

(ii) $U_{\alpha}$ is compact if and only if $\alpha_{\bmod } \in c_{0}\left(l_{\infty}^{n} \mid n \in \mathbb{N}\right)$.

(iii) for $p \geq 2, U_{\alpha}$ is absolutely summing; for $1 \leq p<2, U$ is absolutely summing if and only if $\alpha_{\bmod } \in l_{\infty}\left(l_{r}^{n} \mid n \in \mathbb{N}\right)$.

(iv) for $p \geq 2, U_{\alpha}$ is nuclear if and only if $U_{\alpha}$ is compact if and only if $\alpha_{\text {mod }} \in c_{0}\left(l_{\infty}^{n} \mid n \in \mathbb{N}\right)$; for $1 \leq p<2, U_{\alpha}$ is nuclear if and only if $\alpha_{\bmod } \in$ $c_{0}\left(l_{r}^{n} \mid n \in \mathbb{N}\right)$.

(bb) Let $k \geq 2$ be a natural number, $X$ a Banach space which contains $l_{\infty}^{n}$ 's uniformly, $\left(\alpha_{n i}\right)_{i, n}$ such that $\alpha_{\bmod } \in l_{\infty}\left(l_{\infty}^{n} \mid n \in \mathbb{N}\right)$. Let $U_{\alpha}: C[0,1] \rightarrow c_{0}(X)$ be the operator defined by

$$
U_{\alpha}(f)=\left(\text { Average }_{k}\left(\alpha_{n i} J\left(e_{n i}\right) \int_{0}^{1} f(t) r_{n+i}(t) d t\right) \mid 1 \leq i \leq n\right)_{n \in \mathbb{N}} .
$$

Then

(i) $U_{\alpha}$ is absolutely summing.

(ii) $U_{\alpha}$ is compact if and only if $U_{\alpha}$ is nuclear if and only if $\alpha_{\bmod } \in c_{0}\left(l_{\infty}^{n} \mid n\right.$ $\in \mathbb{N})$.

(cc) Let $k \geq 2$ be a natural number, $X$ a Banach space which contains $l_{\infty}^{n}$ 's uniformly, $\left(\alpha_{n i j}\right)_{i, j, n}$ such that $\beta_{\bmod } \in l_{\infty}\left(l_{\infty}^{n}\left(l_{\infty}^{n}\right) \mid n \in \mathbb{N}\right)$ and for each natural number $n$ denote

$$
x_{n i}=\alpha_{n i 1} J\left(e_{n 1}\right)+\alpha_{n i 2} J\left(e_{n 2}\right)+\cdots+\alpha_{n i n} J\left(e_{n n}\right) .
$$

Let $U_{\beta}: C[0,1] \rightarrow c_{0}(X)$ be the operator defined by

$$
U_{\beta}(f)=\left(\text { Average }_{k}\left(x_{n i} \int_{0}^{1} f(t) r_{n+i}(t) d t\right) \mid 1 \leq i \leq n\right)_{n \in \mathbb{N}} .
$$

Then

(i) $U_{\beta}$ is weakly compact.

(ii) $U_{\beta}$ is compact if and only if $\beta_{\bmod } \in c_{0}\left(l_{\infty}^{n}\left(l_{\infty}^{n}\right) \mid n \in \mathbb{N}\right)$.

(iii) $U_{\beta}$ is absolutely summing if and only if $\beta_{\bmod } \in l_{\infty}\left(l_{\infty}^{n}\left(l_{2}^{n}\right) \mid n \in \mathbb{N}\right)$.

(iv) $U_{\beta}$ is nuclear if and only if $\beta_{\bmod } \in c_{0}\left(l_{\infty}^{n}\left(l_{2}^{n}\right) \mid n \in \mathbb{N}\right)$.

Proof. (a) From Corollary $7(\mathrm{a}), U_{\alpha}$ is weakly compact and further $U_{\alpha}$ is compact if and only if $w_{2}\left(\alpha_{n i} J\left(e_{n i}\right) \mid 1 \leq i \leq n\right) \rightarrow 0$;

$U_{\alpha}$ is absolutely summing if and only if $\sup _{1} w_{1}\left(\alpha_{n i} J\left(e_{n i}\right) \mid 1 \leq i \leq n\right)<\infty$;

$U_{\alpha}$ is nuclear if and only if $w_{1}\left(\alpha_{n i} J\left(e_{n i}\right) \mid 1 \leq i \leq n\right) \rightarrow 0$. 
From $(*)$ and Proposition 8(iii) and (iv) the statement follows.

(aa) The proof is similar to that of (a) and use Corollary 7(b). We omit the details. The proofs of (b), (bb) and (c), (cc) are also similar to that of (a). We prove now (c).

From Corollary $7\left(\right.$ a) $U_{\beta}$ is weakly compact and further

$U_{\beta}$ is compact if and only if $w_{2}\left(x_{n i} \mid 1 \leq i \leq n\right) \rightarrow 0$;

$U_{\beta}$ is absolutely summing if and only if $\sup _{n \in \mathbb{N}} w_{1}\left(x_{n i} \mid 1 \leq i \leq n\right)<\infty$;

$U_{\beta}$ is nuclear if and only if $w_{1}\left(x_{n i} \mid 1 \leq i \leq n\right) \rightarrow 0$.

From $(*)$ an Proposition 8(iii) and (v) we get the statement.

Since, by the famous Dvoretzky theorem, see [2, Chapter 19], each infinite dimensional Banach space contains $l_{2}^{n}$ 's uniformly, i.e., $\forall \varepsilon>0, \forall n \in \mathbb{N}$ there exists a bounded linear operator $J: l_{2}^{n} \rightarrow X$ such that

$$
\|\xi\|_{2} \leq\|J(\xi)\|_{X} \leq(1+\varepsilon)\|\xi\|_{2}, \forall \xi \in l_{2}^{n}
$$

from Example 15(a), (aa) we get the next example; we use in the statement of this example the operator $J$ from $(* *)$.

Example 16. Let $X$ be an infinite dimensional Banach space and $\left(\alpha_{n i}\right)_{i, n}$. by

(a) If $\alpha \in l_{\infty}\left(l_{\infty}^{n} \mid n \in \mathbb{N}\right)$, let $U_{\alpha}: C[0,1] \rightarrow c_{0}(X)$ be the operator defined

$$
U_{\alpha}(f)=\left(\text { Average }\left(\alpha_{n i} J\left(e_{n i}\right) \int_{0}^{1} f(t) r_{n+i}(t) d t\right) \mid 1 \leq i \leq n\right)_{n \in \mathbb{N}} .
$$

Then

(i) $U_{\alpha}$ is weakly compact.

(ii) $U_{\alpha}$ is compact if and only if $\alpha \in c_{0}\left(l_{\infty}^{n} \mid n \in \mathbb{N}\right)$.

(iii) $U_{\alpha}$ is absolutely summing if and only if $\alpha \in l_{\infty}\left(l_{2}^{n} \mid n \in \mathbb{N}\right)$.

(iv) $U_{\alpha}$ is nuclear if and only if $\alpha \in c_{0}\left(l_{2}^{n} \mid n \in \mathbb{N}\right)$.

(b) If $k \geq 2$ is a natural number and $\alpha_{\bmod } \in l_{\infty}\left(l_{\infty}^{n} \mid n \in \mathbb{N}\right)$, let $U_{\alpha}$ : $C[0,1] \rightarrow c_{0}(X)$ be the operator defined by

$$
U_{\alpha}(f)=\left(\text { Average }_{k}\left(\alpha_{n i} J\left(e_{n i}\right) \int_{0}^{1} f(t) r_{n+i}(t) d t\right) \mid 1 \leq i \leq n\right)_{n \in \mathbb{N}} .
$$

Then

(i) $U_{\alpha}$ is absolutely summing.

(ii) $U_{\alpha}$ is nuclear if and only if $U_{\alpha}$ is compact if and only if $\alpha_{\bmod } \in c_{0}\left(l_{\infty}^{n} \mid n \in\right.$ $\mathbb{N})$. (xi).

The proof of the next example follows from Corollary 7 and Proposition 8(x),

Example 17. Let $\left(S_{n}, \Xi_{n}, \nu_{n}\right)_{n \in \mathbb{N}}$ be a sequence of finite measure spaces.

(a) Let $\left(g_{n i}\right)_{1 \leq i \leq n} \subset L_{1}\left(\nu_{n}\right), g_{n}=\left(g_{n 1}, \ldots, g_{n n}\right): S_{n} \rightarrow \mathbb{K}^{n}$ be such that

$$
\sup _{n \in \mathbb{N}} \sup _{\beta \in l_{2}^{n},\|\beta\|_{2} \leq 1} \int_{S_{n}}\left\|M_{\beta}\left(g_{n}\left(s_{n}\right)\right)\right\|_{2} d \nu_{n}\left(s_{n}\right)<\infty \text {. }
$$


AVERAGES, COMPACT, ABSOLUTELY SUMMING AND NUCLEAR OPERATORS 923

Let $U: C[0,1] \rightarrow c_{0}\left(L_{1}\left(\lambda \otimes \nu_{n}\right) \mid n \in \mathbb{N}\right)$ be the operator defined by

$$
U(f)=\left(\text { Average }\left(r_{i} g_{n i} \int_{0}^{1} f(t) r_{n+i}(t) d t \mid 1 \leq i \leq n\right) ; L_{1}\left(\lambda \otimes \nu_{n}\right)\right)_{n \in \mathbb{N}} .
$$

Then

(i) $U$ is weakly compact.

(ii) $U$ is compact if and only if

$$
\sup _{\beta \in l_{2}^{n},\|\beta\|_{2} \leq 1} \int_{S_{n}}\left\|M_{\beta}\left(g_{n}\left(s_{n}\right)\right)\right\|_{2} d \nu_{n}\left(s_{n}\right) \rightarrow 0 .
$$

(iii) $U$ is absolutely summing if and only if $\sup _{n \in \mathbb{N}} \int_{S_{n}}\left\|g_{n}\left(s_{n}\right)\right\|_{2} d \nu_{n}\left(s_{n}\right)<\infty$.

(iv) $U$ is nuclear if and only if $\int_{S_{n}}\left\|g_{n}\left(s_{n}\right)\right\|_{2} d \nu_{n}\left(s_{n}\right) \rightarrow 0$.

(b) Let $\left(\alpha_{n i}\right)_{i, n} \subset \mathbb{K},\left(E_{n i}\right)_{1 \leq i \leq n} \subset \Sigma_{n}$ be a partition for $S_{n}$,

$$
\beta_{n}=\left(\alpha_{n 1} \nu_{n}\left(E_{n 1}\right), \ldots, \alpha_{n n} \nu_{n}\left(E_{n n}\right)\right), \beta=\left(\beta_{n}\right)_{n \in \mathbb{N}}
$$

such that $\beta \in l_{\infty}\left(l_{2}^{n} \mid n \in \mathbb{N}\right)$.

Let $U: C[0,1] \rightarrow c_{0}\left(L_{1}\left(\lambda \otimes \nu_{n}\right) \mid n \in \mathbb{N}\right)$ be the operator defined by

$$
U(f)=\left(\text { Average }\left(\alpha_{n i} r_{i} \chi_{E_{n i}} \int_{0}^{1} f(t) r_{n+i}(t) d t \mid 1 \leq i \leq n\right) ; L_{1}\left(\lambda \otimes \nu_{n}\right)\right)_{n \in \mathbb{N}} .
$$

Then

(i) $U$ is weakly compact.

(ii) $U$ is compact if and only if $\beta \in c_{0}\left(l_{2}^{n} \mid n \in \mathbb{N}\right)$.

(iii) $U$ is absolutely summing if and only if $\beta \in l_{\infty}\left(l_{1}^{n} \mid n \in \mathbb{N}\right)$.

(iv) $U$ is nuclear if and only if $\beta \in c_{0}\left(l_{1}^{n} \mid n \in \mathbb{N}\right)$.

(bb) Let $k \geq 2$ be a natural number, $\left(\alpha_{n i}\right)_{i, n} \subset \mathbb{K},\left(E_{n i}\right)_{1 \leq i \leq n} \subset \Sigma_{n}$ be a partition for $S_{n}, \beta_{n}=\left(\alpha_{n 1} \nu_{n}\left(E_{n 1}\right), \ldots, \alpha_{n n} \nu_{n}\left(E_{n n}\right)\right), \beta_{\bmod }=\left(b_{n k} \beta_{n}\right)_{n \in \mathbb{N}}$ such that $\beta_{\text {mod }} \in l_{\infty}\left(l_{\infty}^{n} \mid n \in \mathbb{N}\right)$.

Let $U: C[0,1] \rightarrow c_{0}\left(L_{1}\left(\mu \otimes \nu_{n}\right) \mid n \in \mathbb{N}\right)$ be the operator defined by

$$
U(f)=\left(\text { Average }_{k}\left(\alpha_{n i} r_{i} \chi_{E_{n i}} \int_{0}^{1} f(t) r_{n+i}(t) d t \mid 1 \leq i \leq n\right) ; L_{1}\left(\lambda \otimes \nu_{n}\right)\right)_{n \in \mathbb{N}} .
$$

Then

(i) $U$ is weakly compact.

(ii) $U$ is compact if and only if $\beta_{\text {mod }} \in c_{0}\left(l_{\infty}^{n} \mid n \in \mathbb{N}\right)$.

(iii) $U$ is absolutely summing if and only if $\beta_{\bmod } \in l_{\infty}\left(l_{2}^{n} \mid n \in \mathbb{N}\right)$.

(iv) $U$ is nuclear if and only if $\beta_{\bmod } \in c_{0}\left(l_{2}^{n} \mid n \in \mathbb{N}\right)$.

Acknowledgements. We would like to express our gratitude to the referee for many valuable comments, suggestions which have improved the final version of the paper. 


\section{References}

[1] A. Defant and K. Floret, Tensor Norms and Operators Ideals, North-Holland Mathematics Studies, 176. North-Holland Publishing Co., Amsterdam, 1993.

[2] J. Diestel, H. Jarchow, and A. Tonge, Absolutely Summing Operators, Cambridge Studies in Advanced Mathematics, 43. Cambridge University Press, Cambridge, 1995.

[3] J. Diestel and J. J. Uhl, Vector Measures, Mathematical Surveys, No. 15. American Mathematical Society, Providence, R.I., 1977.

[4] L. Drewnowski and Z. Lipecki, On vector measures which have everywhere infinite variation or noncompact range, Dissertationes Math. (Rozprawy Mat.) 339 (1995), 39 pp.

[5] R. Latała and K. Oleszkiewicz, On the best constant in the Khinchin-Kahane inequality, Studia Math. 109 (1994), no. 1, 101-104.

[6] A. Pietsch, Eigenvalues and s-numbers, Akademische Verlagsgesellschaft Geest \& Portig K.-G., Leipzig, 1987.

[7] A. Pietsch and J. Wenzel, Orthonormal Systems and Banach Space Geometry, Encyclopedia of Mathematics and its Applications, 70. Cambridge University Press, Cambridge, 1998.

[8] D. Popa, Examples of operators on $C[0,1]$ distinguishing certain operator ideals, Arch. Math. (Basel) 88 (2007), no. 4, 349-357.

[9] N. Tomczak-Jagermann, Banach-Mazur distances and finite dimensional operator ideals, Pitman Monographs and Surveys in Pure and Applied Mathematics, vol. 38, Harlow: Longman Scientific \& Technical; New York: John Wiley \& Sons, Inc., 1989.

Department of Mathematics

UNIVERSITY OF CONSTANTA

Bd. Mamaia 124, 8700 Constanta, Romania

E-mail address: dpopa@univ-ovidius.ro 